\title{
Quantum teleportation and entanglement swapping of matter qubits with coherent multiphoton states
}

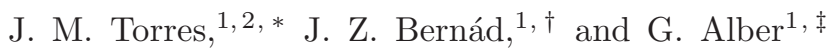 \\ ${ }^{1}$ Institut für Angewandte Physik, Technische Universität Darmstadt, D-64289, Germany \\ ${ }^{2}$ Departamento de Investigación en Física, Universidad de Sonora, Hermosillo, México
}

(Dated: June 13, 2021)

\begin{abstract}
Protocols for probabilistic entanglement-assisted quantum teleportation and for entanglement swapping of material qubits are presented. They are based on a protocol for postselective Bellstate projection which is capable of projecting two material qubits onto a Bell state with the help of ancillary coherent multiphoton states and postselection by balanced homodyne photodetection. Provided this photonic postselection is successful we explore the theoretical possibilities of realizing unit fidelity quantum teleportation and entanglement swapping with $25 \%$ success probability. This photon-assisted Bell projection is generated by coupling almost resonantly the two material qubits to single modes of the radiation field in two separate cavities in a Ramsey-type interaction sequence and by measuring the emerged field states in a balanced homodyne detection scenario. As these quantum protocols require basic tools of quantum state engineering of coherent multiphoton states and balanced homodyne photodetection they may offer interesting perspectives in particular for current quantum optical applications in quantum information processing.
\end{abstract}

PACS numbers: 03.67.Bg, 03.67.Hk, 42.50.Ct

\section{INTRODUCTION}

The development of physical procedures for the establishment of entanglement between distant material quantum systems, such as qubits, capable of storing quantum information reliably is an important prerequisite for quantum communication [1]. Such material quantum systems may form the nodes of a quantum network [2], for example, which are possibly also connected by photonic channels enabling the direct transfer of quantum information or the establishment of entanglement. However, as typically direct transfer of quantum information over photonic channels is affected by loss processes and by decoherence it may be advantageous to exploit already existing entanglement between nodes within such a network for purposes of reliable exchange of quantum information. Furthermore, controlled redistribution of entanglement within such a quantum network may be used to establish new routes for exchange of quantum information. Reliable transfer of quantum information may be achieved with the help of entanglement-enabled quantum teleportation [3] and redistribution of entanglement with the help of entanglement swapping. In order to be able to realize these two important elementary quantum information processing protocols in material qubit systems it is necessary to implement projective Bell measurements which can be performed reliably locally at each node of such a quantum network. Complete Bell measurements capable of distinguishing all four Bell states are still difficult to realize experimentally. In view of these consid-

\footnotetext{
*Electronic address: Mauricio.Torres@physik.tu-darmstadt.de

${ }^{\dagger}$ Electronic address: Zsolt.Bernad@physik.tu-darmstadt.de

‡Electronic address: gernot.alber@physik.tu-darmstadt.de
}

erable experimental difficulties it is of current interest to develop implementations of perfect postselective Bell projections. In such a projective Bell measurement two material qubits are projected onto a particular Bell state probabilistically in such a way that, provided this projective measurement is successful, this two-qubit Bell state is postselected with unit fidelity.

Recently, several proposals have been made for implementing a quantum repeater [4] which redistributes entanglement from intermediary entangled material qubit pairs to distant qubits with the help of entanglement swapping [5 8]. Thereby imperfections affecting the entanglement swapping can be compensated afterwards by entanglement purification [9 11].

First physical implementations of entanglementassisted quantum teleportation were realized with photonic qubits 12 14. Subsequent experiments achieved teleportation over distances of $100 \mathrm{~km} \mathrm{[15,} \mathrm{16].} \mathrm{First}$ experiments on teleportation with material qubits were limited to distances of the order of a few $\mu \mathrm{m}$ [17, 18]. However, most recent realizations report successful teleportation with material qubits over distances of $21 \mathrm{~m}$ [19] with the help of ancillary photon exchange.

Despite these recent experimental advances these realizations of quantum information transfer are limited to distances of the order of $100 \mathrm{~km}$ mainly due to the use of single or few photon states acting as ancillary quantum systems. In order to overcome this hurdle coherent photon states offer interesting perspectives. Techniques for their generation, manipulation and detection are well developed and these multiphoton states of the radiation field can be transmitted in a controlled way through already existing optical communication networks. The hybrid quantum repeater model of van Loock et al. [20] is an early example which aims at exploiting these advantages of coherent multiphoton states for purposes of 
quantum information processing.

Motivated by these advantages and by the fundamental role played by Bell-state projections in basic quantum communication protocols in this paper we propose a protocol for implementing a probabilistic Bell-state projection of material qubits with the help of coherent multiphoton states and of photonic postselection by balanced homodyne photodetection. This postselective measurement protocol results in a Bell state with almost unit fidelity and success probability depending on the overlap of the initial material state with this specific Bell state. In our scenario single modes of the radiation field initially prepared in coherent states are used as ancillary quantum systems in a Ramsey-type interaction sequence. These photonic states have a specific phase difference and interact almost resonantly with the two qubits for appropriately chosen interaction times. Built on this procedure we propose probabilistic protocols for entanglement-assisted quantum teleportation and for entanglement swapping.

The probabilistic photon-assisted Bell projection discussed in this paper is based on two crucial dynamical properties. Firstly, it takes advantage of a characteristic property of the two-qubit Tavis-Cummings model 21] describing the almost resonant interaction between two qubits and a single mode of the radiation field, namely the existence of an invariant two-qubit Bell state which is not coupled to the photons. However, this characteristic property with the aid of a photonic postselection can generate an almost perfect Bell state only for specific initial conditions. In the case of arbitrary initial conditions the two-qubit quantum states resulting from a photonic postselection are noisy Bell states. It is shown that the elimination of these noisy contributions can be achieved by the second essential dynamical property of our protocol, namely the involvement of a Ramsey-type interaction scenario.

This paper is organized as follows. In Sec I the quantum electrodynamical interaction between two material qubits and a single mode of the radiation field is discussed within the framework of the Tavis-Cummings 21] model. Approximate analytical solutions are presented for the time evolution of the entangled matter-photon quantum state which are valid for initially prepared coherent field states and for almost resonant interaction between the two qubits and the photons. Furthermore, a detailed discussion of the two-qubit quantum state is presented which results from photonic postselection by balanced homodyne detection. In Sec. III these results are generalized to a Ramsey-type interaction scenario involving two subsequent matter-field interactions in two cavities and two photonic postselection processes by balanced homodyne detection. It is shown that this procedure can prepare a Bell state with unit fidelity for any given initial condition of the two material qubits and with success probability given by the initial probability weight of the generated Bell state. In Sec. IV we discuss effects that arise from unequal coupling strengths of the qubits to the radiation field and from different transition frequencies of the qubits. Finally, in Sec. $\nabla$ implementations of entanglement-assisted quantum teleportation and entanglement swapping are discussed which are based on the postselective Bell-state projection of Sec. III] A detailed derivation of the solution of the two-qubit TavisCummings model is given in Appendix A. For the sake of completeness in Appendix B basic facts concerning the theoretical description of balanced homodyne photodetection are summarized. In Appendix $\mathbb{C}$ we include analytical calculations that support Sec. IV.

\section{THE TWO-QUBIT TAVIS-CUMMINGS MODEL}

In this section we discuss basic dynamical features of the two-qubit Tavis-Cummings model [21]. This model describes the interaction between two two-level systems (material qubits) and a single-mode of the radiation field inside a cavity. As this model involves an interactioninsensitive two-qubit Bell state it is possible to prepare this maximally entangled two-qubit state by projection onto an appropriate photonic quantum state. For initially prepared coherent states of the radiation field this projection can be achieved by postselection with the help of balanced homodyne photodetection.

\section{A. The qubit-field dynamics}

We consider two two-level systems (material qubits), say $A$ and $B$, with ground states $|0\rangle_{i}$ and excited states $|1\rangle_{i}(i \in\{A, B\})$ separated by an energy difference $\hbar \omega_{\mathrm{a}}$ from their ground states. Both two-level systems are assumed to have equal transition dipole moments between the almost resonantly coupled energy eigenstates $|0\rangle_{i}$ and $|1\rangle_{i}$ of different parity. In the dipole and rotating-wave approximation the two-qubit Tavis-Cummings Hamiltonian describing almost resonant interaction of these two qubits with a single mode of the radiation field is given by

$$
\hat{H}=\hbar \omega \hat{a}^{\dagger} \hat{a}+\sum_{i=A, B} \hbar\left(\frac{\omega_{\mathrm{a}}}{2} \hat{\sigma}_{i}^{z}+g \mathrm{e}^{i \theta} \hat{\sigma}_{i}^{+} \hat{a}+g \mathrm{e}^{-i \theta} \hat{\sigma}_{i}^{-} \hat{a}^{\dagger}\right)
$$

with $\hat{\sigma}_{i}^{z}=|1\rangle\left\langle\left. 1\right|_{i}-\mid 0\right\rangle\left\langle\left. 0\right|_{i}(i \in\{A, B\})\right.$. The ladder operators of the qubits are denoted by $\hat{\sigma}_{i}^{+}=|1\rangle\left\langle\left. 0\right|_{i}\right.$ and $\hat{\sigma}_{i}^{-}=|0\rangle\left\langle\left. 1\right|_{i}\right.$ and the radiative coupling of the qubits to the single mode of the radiation field is characterized by the vacuum Rabi frequency $2 g$ and the phase $\theta$. The annihilation and creation operators of the single-mode radiation field with frequency $\omega$ are denoted by $\hat{a}$ and $\hat{a}^{\dagger}$. The detuning between the radiation field and the transition frequency of the two-level systems is given by $\delta=\omega_{a}-\omega$.

In our subsequent discussion we are particularly interested in solutions of the time dependent Schrödinger 
equation governed by the Hamiltonian of Eq. (11). We assume that initially the matter-field system is prepared in a pure separable quantum state

$$
\left|\Psi_{0}\right\rangle=\left(c_{-}\left|\Psi^{-}\right\rangle+c_{1}|1,1\rangle+c_{+}\left|\Psi^{+}\right\rangle+c_{0}|0,0\rangle\right)|\alpha\rangle,
$$

where the pure two-qubit state is expanded in the orthonormal Bell states

$$
\left|\Psi^{ \pm}\right\rangle=\frac{1}{\sqrt{2}}(|0,1\rangle \pm|1,0\rangle)
$$

and the separable states $|1,1\rangle$ and $|0,0\rangle$ with $|i\rangle_{A}|j\rangle_{B}=$ $|i, j\rangle(i, j \in\{0,1\})$. The single mode of the radiation field is in a coherent state

$$
|\alpha\rangle=\sum_{n=0}^{\infty} \mathrm{e}^{-\frac{|\alpha|^{2}}{2}} \frac{\alpha^{n}}{\sqrt{n !}}|n\rangle, \quad \alpha=\sqrt{\bar{n}} \mathrm{e}^{i \phi}
$$

with phase $\phi$, mean photon number $\bar{n}$ and $|n\rangle\left(n \in \mathbb{N}_{0}\right)$ denoting the normalized photon-number states. Normalization of $\left|\Psi_{0}\right\rangle$ requires the condition $\left|c_{-}\right|^{2}+\left|c_{+}\right|^{2}+\left|c_{0}\right|^{2}+$ $\left|c_{1}\right|^{2}=1$.

In the following we shall take advantage of a special feature of the two-qubit Tavis Cummings model, namely that quantum states of the form $\left|\Psi^{-}\right\rangle|n\rangle\left(n \in \mathbb{N}_{0}\right)$ with the photon-number state $|n\rangle$ are stationary eigenstates of the Hamiltonian of Eq. (1) with eigenvalue $\hbar \omega n$.

The time evolution of an initial state of the form of Eq. (2) can be obtained from the solution of the eigenvalue problem of the two-qubit Tavis-Cummings Hamiltonian (1). This solution is presented in detail in Appendix A Here, we merely present the final result of the time dependent tripartite quantum state, i.e.

$$
\begin{aligned}
|\Psi(t)\rangle & =c_{-}\left|\Psi^{-}\right\rangle\left|\alpha \mathrm{e}^{-i \omega t}\right\rangle+ \\
& +|1,1\rangle\left|\chi_{1}(t)\right\rangle+\left|\Psi^{+}\right\rangle\left|\chi_{0}(t)\right\rangle+|0,0\rangle\left|\chi_{-1}(t)\right\rangle
\end{aligned}
$$

The matter-field state $|\Psi(t)\rangle$ displays the interaction between the material systems $A, B$ and the single-mode of the radiation field. According to Eq. (5) the coherent state $\left|\alpha \mathrm{e}^{-i \omega t}\right\rangle$ is strictly correlated with the maximally entangled material Bell state $\left|\Psi^{-}\right\rangle$. Therefore, if we were able to discriminate the field state $\left|\alpha \mathrm{e}^{-i \omega t}\right\rangle$ from the other three field states $\left|\chi_{j}(t)\right\rangle(j \in\{-1,0,1\})$ we could prepare the maximally entangled material Bell state $\left|\Psi^{-}\right\rangle$ in a probabilistic way. However, this discrimination of the field states is not a straightforward task as they are not orthogonal, in general, so that they cannot be distinguished perfectly. For our subsequent development of a probabilistic scheme for entanglement swapping and quantum teleportation based on coherent field states and photonic postselection by homodyning it will be of crucial importance to be able to distinguish these field states almost perfectly.

Some basic properties of the pure field states which determine the tripartite quantum state $|\Psi(t)\rangle$ can be studied by considering the time dependence of the overlaps between the coherent state $\left|\alpha \mathrm{e}^{-i \omega t}\right\rangle$ and the other three field states $\left|\chi_{j}(t)\right\rangle(j \in\{-1,0,1\})$ as depicted in Fig.

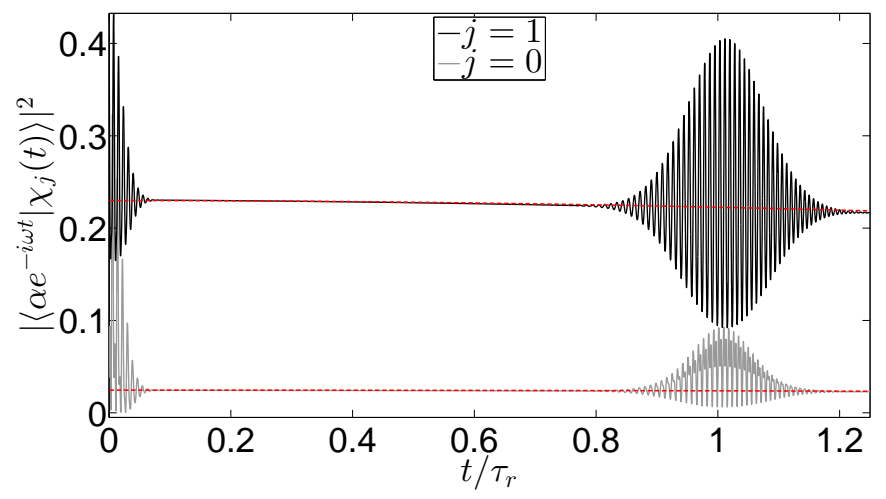

FIG. 1: Overlap between the exact photonic states $\left|\chi_{j}(t)\right\rangle$ (see Eq. (5)) and the coherent state $\left|\alpha \mathrm{e}^{-i \omega t}\right\rangle$ showing the collapse and revival phenomena. Typically, in the collapse region the overlap is nonzero and proportional to the parameter $|\eta(\vec{c}, \phi)|^{2}$ of Eq. (10). The upper and lower lines (red) show the approximation given in Eq. 13). The overlap for $j=-1$ is not shown and behaves qualitatively as for $j=1$. The parameters are $\alpha=7.6 \mathrm{e}^{i 2.65}, c_{-}=0.5446 \mathrm{e}^{i}, c_{1}=0.6389 \mathrm{e}^{-i 1.8}, c_{+}=$ $0.1950 \mathrm{e}^{-i 0.3}, c_{0}=0.5071 \mathrm{e}^{i 1.3}, \delta / g=3.5$, and $\theta=0$.

1. These overlaps resemble collapse and revival phenomena which also appear in a similar form in the JaynesCummings model 22]. After a collapse time $\tau_{c}$ initial rapid oscillations of the overlaps decay to a 'plateau' characterized by an almost constant value. After a revival time $\tau_{r}$ the rapid oscillations reappear. Thus, a perfect discrimination of the material Bell state $\left|\Psi^{-}\right\rangle$from the other material quantum states $|0,0\rangle$ and $|1,1\rangle$ would be possible in the plateau region if these overlaps vanished. However, such vanishing overlaps in the plateau region can only be achieved for very special initial conditions of the two qubits as will be demonstrated in the following.

In order to gain insight into the intricate dynamical evolution of $|\Psi(t)\rangle$ let us concentrate on the case of large mean photon numbers. For initial field states $|\alpha\rangle$ with $\bar{n} \gg 1$ it is possible to simplify the time dependent solution $|\Psi(t)\rangle$ of Eq.(5) significantly by expanding the eigenvalues of the Tavis-Cummings Hamiltonian around $\bar{n}$ up to first order in $n$, i.e.

$$
E_{j}^{(n)} \approx \hbar\left[\Delta_{j}+\left(\omega+\varpi_{j}\right)(n-1)\right]
$$

with

$$
\begin{aligned}
& \Delta_{j}=j \frac{2 g^{2} \bar{n}+\delta^{2}}{\Omega_{\bar{n}}}+\delta g^{2} \frac{\omega_{\bar{n}}^{2}+\Omega_{\bar{n}}^{2}-2 g^{2}}{\Omega_{\bar{n}}^{4}}(-1)^{j} 2^{\delta_{j, 0}}, \\
& \varpi_{j}=j \frac{2 g^{2}}{\Omega_{\bar{n}}}-\frac{4 \delta}{\left(\Omega_{\bar{n}} / g\right)^{4}}(-1)^{j} 2^{\delta_{j, 0}} \\
& \omega_{n}=g \sqrt{4 n-2}, \quad \Omega_{n}=\sqrt{g^{2}(4 n-2)+\delta^{2}}
\end{aligned}
$$

and with the Kronecker delta $\delta_{i, j}$. The index $j=-1,0,1$ distinguishes the three eigenvalues of each coupled block with photon number $n$. Depending on whether $|j|=1$ or 
$j=0$ the frequency $\Delta_{j}$ introduces two largely different time scales because in the limit $\bar{n} \gg 1$ we obtain the result $\Delta_{ \pm 1} / \Delta_{0} \sim \varpi_{ \pm 1} / \varpi_{0} \sim 3 \bar{n}$. According to the first order expansion the validity of Eq. (6) is restricted to times $\tau$ with

$$
\frac{1}{2 \hbar}\left|\frac{d^{2} E_{j}^{(n)}}{d n^{2}}\right|_{n=\bar{n}} \tau \bar{n} \ll 2 \pi .
$$

In this approximation the field states can be written as a superposition of coherent states, i.e.

$$
\left|\chi_{j}(t)\right\rangle=\sum_{k=-1}^{1} \eta_{j, k} \mathrm{e}^{i\left[j\left(\phi+\theta-\left(\omega+\varpi_{k}\right) t\right)-\Delta_{k} t\right]}\left|\alpha \mathrm{e}^{-i\left(\omega+\varpi_{k}\right) t}\right\rangle
$$

with the parameters

$$
\begin{gathered}
\eta_{j, 0}=\left(\frac{\delta}{\omega_{\bar{n}}}\right)^{\delta_{j, 0}} \frac{(-1)^{\delta_{j, 1}}}{\sqrt{2^{|j|}}} \eta(\vec{c}, \phi), \quad \vec{c}=\left(c_{+}, c_{0}, c_{1}\right), \\
\eta(\vec{c}, \phi)=\frac{\omega_{\bar{n}}^{2}}{\Omega_{\bar{n}}^{2}}\left(\frac{\delta}{\omega_{\bar{n}}} c_{+}+\frac{c_{0} \mathrm{e}^{i(\phi+\theta)}-c_{1} \mathrm{e}^{-i(\phi+\theta)}}{\sqrt{2}}\right), \\
\eta_{j, \pm 1}=\left(\frac{\delta \pm \Omega_{\bar{n}}}{\omega_{\bar{n}}}\right)^{j} \frac{\omega_{\bar{n}}^{2}}{\sqrt{2^{|j|}} 2 \Omega_{\bar{n}}^{2}} \times \\
\left(c_{+}+\frac{\omega_{\bar{n}} c_{0} \mathrm{e}^{i(\phi+\theta)}}{\sqrt{2}\left(\delta \pm \Omega_{\bar{n}}\right)}+\frac{\left(\delta \pm \Omega_{\bar{n}}\right) c_{1} \mathrm{e}^{-i(\phi+\theta)}}{\sqrt{2} \omega_{\bar{n}}}\right) .
\end{gathered}
$$

These approximate solutions of the field states yield further insight into the collapse and revival phenomena apparent in Fig. 11 as these overlaps are determined by

$$
\begin{aligned}
\left\langle\alpha \mathrm{e}^{-i \omega t} \mid \alpha \mathrm{e}^{-i\left(\omega+\varpi_{j}\right) t}\right\rangle & =\mathrm{e}^{-\bar{n}\left(1-\mathrm{e}^{-i \varpi_{j} t}\right)} . \\
& \approx \mathrm{e}^{-\bar{n}\left(i \varpi_{j} t+\varpi_{j} t^{2} / 2\right)} .
\end{aligned}
$$

An additional approximation of the last line is valid only for short times $t$ with $t \ll 2 \pi / \varpi_{j}$. In order to meet the requirement of condition (8) we have to restrict our description to the shortest time scale or highest frequencies $\varpi_{ \pm 1}$. These two frequencies are of the same order and characterize time scales of the collapse and the revival phenomena. The revival time $\tau_{r}$ is characterized by a vanishing exponent in the first line of Eq. (11). The exponential decay in the second line defines the collapse time $\tau_{c}$. Accordingly, these two characteristic times are given by

$$
\tau_{r}=\frac{\pi}{g} \sqrt{4 \bar{n}-2+\frac{\delta^{2}}{g^{2}}}, \quad \tau_{c}=\frac{\tau_{r}}{\pi \sqrt{2 \bar{n}}} .
$$

Therefore, for interaction times $\tau$ in the plateau region of Fig. 1, i.e. $\tau_{c}<\tau \ll \tau_{r}$ the relevant overlaps between the field states can be approximated by

$$
\left|\left\langle\alpha \mathrm{e}^{-i \omega \tau} \mid \chi_{j}(\tau)\right\rangle\right|^{2}=\frac{\mathrm{e}^{-\bar{n} \varpi_{0}^{2} \tau^{2}}}{2^{|j|}}\left(\frac{\delta}{\omega_{\bar{n}}}\right)^{2 \delta_{j, 0}}|\eta(\vec{c}, \phi)|^{2} .
$$

From Eq. (13) it is apparent that in the plateau region the three relevant overlaps are proportional to the parameter $\eta(\vec{c}, \phi)$ of Eq. (10). The overlap between $\left|\alpha \mathrm{e}^{-i \omega t}\right\rangle$ and $\left|\chi_{0}(t)\right\rangle$ is the only one which is proportional to the detuning $\delta$. Therefore, for interaction times $\tau_{c} \ll \tau \ll \tau_{r}$ the state $\left|\chi_{0}(t)\right\rangle$ is always orthogonal to the free coherent state $\left|\alpha \mathrm{e}^{-i \omega t}\right\rangle$ provided the interaction between the twolevel systems and the single mode of the radiation field is resonant, i.e. $\delta=0$.

Let us now consider a projective field measurement of the coherent state $\left|e^{-i \omega \tau} \alpha\right\rangle$. The time evolution of the tripartite system is given by Eqs. (5) and (9). For interaction times in the plateau region of Fig. 11, i.e. $\tau_{c} \ll \tau \ll \tau_{r}$, we obtain as a result of such a projective field measurement the unnormalized two-qubit quantum state

$$
\left\langle\alpha \mathrm{e}^{-i \omega \tau}\left|\mathrm{e}^{-i \hat{H} \tau / \hbar}\right| \Psi_{0}\right\rangle=c_{-}\left|\Psi^{-}\right\rangle+\eta(\vec{c}, \phi) s\left|\psi_{\phi}\right\rangle
$$

with

$$
s=\mathrm{e}^{-i\left(\Delta_{0}+\varpi_{0} \bar{n}\right) \tau-\bar{n} \varpi_{0}^{2} \tau^{2} / 2} .
$$

This material quantum state is a superposition of the antisymmetric Bell state $\left|\Psi^{-}\right\rangle$and the unnormalized state

$$
\left|\psi_{\phi}\right\rangle=\frac{\delta}{\omega_{\bar{n}}}\left|\Psi^{+}\right\rangle-\frac{\mathrm{e}^{i(\Theta+\phi)}}{\sqrt{2}}|1,1\rangle+\frac{\mathrm{e}^{-i(\Theta+\phi)}}{\sqrt{2}}|0,0\rangle,
$$

where we have introduced the phase $\Theta=\theta-\left(\omega+\varpi_{0}\right) \tau$. The parameter $\eta(\vec{c}, \phi)$ is given by Eq. (10). The normalization of the state after the projection as given by Eq. (14) yields the success probability $P$ of the projective field measurement, i.e.

$$
P=\left|c_{-}\right|^{2}+|\eta(\vec{c}, \phi)|^{2}\left(1+\frac{\delta^{2}}{\omega_{\frac{2}{n}}^{2}}\right) \mathrm{e}^{-\bar{n} \varpi_{0}^{2} \tau^{2}} .
$$

Thus, perfect projection onto the antisymmetric Bell state $\left|\Psi^{-}\right\rangle$can be achieved by projection onto the coherent state $\left|\alpha \mathrm{e}^{-i \omega \tau}\right\rangle$ only for those special initial conditions for which $\eta(\vec{c}, \phi)$ vanishes, such as perfect resonant interaction $(\delta=0)$, equal initial weights $\left(c_{0}=c_{1}\right)$, and perfectly matched phases $(\phi=-\theta)$. A major challenge of our subsequent discussion will be the development of a photonic measurement scheme by which such a perfect projection can be achieved by this type of photonic postselection for all initial conditions of the form of Eq. (2). In the subsequent section it will be demonstrated that with the help of a Ramsey-type interaction scenario which involves the two material qubits interacting with the modes of two different cavities a material Bell state $\left|\Psi^{-}\right\rangle$can be generated with almost unit fidelity and success probability $\left|c_{-}\right|^{2}$ (see Eq. (2)).

\section{B. Photonic postselection by balanced homodyne detection}

Postselective projection of the tripartite quantum state $|\Psi(\tau)\rangle$ of Eq. (5) onto the coherent state $\left|\alpha e^{-i \omega \tau}\right\rangle$ can be achieved in a convenient way with the help of balanced homodyne detection. As discussed in more detail 
in Appendix B in a typical balanced homodyne detection measurement 23] the single-mode field state to be measured is superposed coherently with an intense coherent state $\| \alpha_{L}\left|e^{i \theta_{L}}\right\rangle$ of a local oscillator by a $50 \%$ reflecting beam splitter and the difference of photon numbers $n_{-}$of the two modes emerging from the beam splitter is measured. If the mode to be measured is prepared in the quantum state $\hat{\rho}_{F}$ 24], the local oscillator state is intense, i.e. $\left|\alpha_{L}\right| \gg 1$, and the homodyne detection is performed with unit quantum efficiency, the detection scheme is equivalent to a projective von Neumann measurement. In particular, the probability of detecting a difference photon number $n_{-}$is given by

$$
P_{\theta_{L}}\left(\frac{n_{-}}{\sqrt{2}\left|\alpha_{L}\right|}\right)=\operatorname{Tr}\left\{\hat{\rho}_{F}\left|q_{\theta_{L}}\right\rangle\left\langle q_{\theta_{L}}\right|\right\}
$$

with the quadrature eigenstate $\left|q_{\theta_{L}}\right\rangle$ being determined by the eigenvalue equation

$$
\frac{1}{\sqrt{2}}\left(\hat{a} e^{-i \theta_{L}}+\hat{a}^{\dagger} e^{i \theta_{L}}\right)\left|q_{\theta_{L}}\right\rangle=q_{\theta_{L}}\left|q_{\theta_{L}}\right\rangle
$$

with the eigenvalues $q_{\theta_{L}} \in \mathbb{R}$ and with $a\left(a^{\dagger}\right)$ denoting the annihilation (creation) operator of the mode to be measured. Thus, in this limit a homodyne detection measurement is a von Neumann measurement determined by the continuous set of orthonormal projectors $\hat{\pi}\left(q_{\theta_{L}}\right)=\left|q_{\theta_{L}}\right\rangle\left\langle q_{\theta_{L}}\right|$. This implies that a postselective photonic measurement with the phase $\theta_{L}=\phi-\omega \tau$ in an interval $q_{\theta_{L}} \in\left(\sqrt{2}|\alpha|-\delta_{L}, \sqrt{2}|\alpha|+\delta_{L}\right)$ projects the field state $\hat{\rho}_{F}=\operatorname{Tr}_{A, B}\{|\Psi(\tau)\rangle\langle\Psi(\tau)|\}$ onto the coherent state $\left|\alpha e^{-i \omega \tau}\right\rangle\left(\alpha=|\alpha| e^{i \phi}\right)$ with almost unit probability provided the interval $\delta_{L}$ is chosen sufficiently large (compare with Eq. (B6) and the estimates of Appendix B).

The Wigner phase space distribution is a convenient way to visualize the field state (compare with Eq. (5))

$$
\hat{\rho}_{F}=\left|c_{-}\right|^{2}\left|\alpha \mathrm{e}^{-i \omega \tau}\right\rangle\left\langle\alpha \mathrm{e}^{-i \omega \tau}\left|+\sum_{j=-1}^{1}\right| \chi_{j}(\tau)\right\rangle\left\langle\chi_{j}(\tau)\right|
$$

emerging from the interaction between the two material quantum systems and the single-mode of the radiation field. It is defined by [25]

$$
W\left(\beta, \beta^{*}\right)=\frac{1}{\pi^{2}} \int \operatorname{Tr}\left\{\hat{\rho}_{F} \mathrm{e}^{\zeta \hat{a}^{\dagger}-\zeta^{*} \hat{a}}\right\} \mathrm{e}^{\beta \zeta^{*}-\beta^{*} \zeta} d^{2} \zeta
$$

with $\beta, \zeta \in \mathbb{C}$. According to Eq. (2) initially, i.e. at $\tau=0, \hat{\rho}_{F}=|\alpha\rangle\langle\alpha|$ is a coherent state so that its Wigner phase space distribution is given by a Gaussian distribution. For $\tau>0$ the mixed field state $\hat{\rho}_{F}$ always contains an admixture of the coherent state $\left|\alpha \mathrm{e}^{-i \omega \tau}\right\rangle$ which is strictly correlated with the material Bell state $\left|\Psi^{-}\right\rangle$ of the two qubits. The free time evolution of this coherent state reflects the fact that the Bell state $\left|\Psi^{-}\right\rangle$ is not coupled to the single-mode radiation field. However, due to the fact that in general $|\Psi(\tau)\rangle$ of Eq. (5) is a tripartite entangled state the Wigner distribution of $\hat{\rho}_{F}$ contains also additional maxima with interference

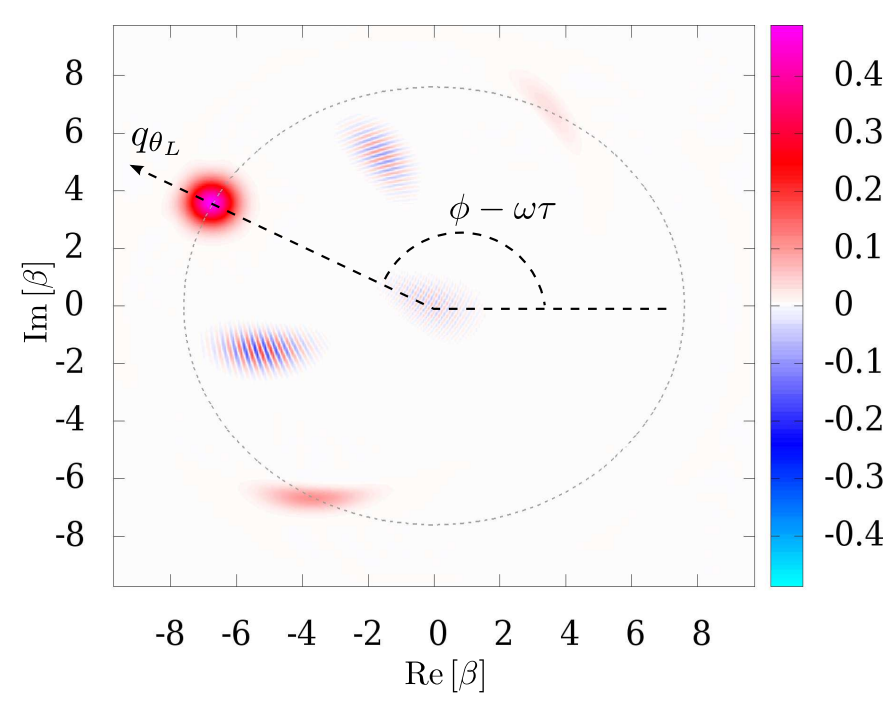

FIG. 2: Wigner phase space distribution of the photonic state $\hat{\rho}_{F}$ : The material state $\left|\Psi^{-}\right\rangle$is solely paired with the Gaussian peak. The rest of the states in Eq. (5) have contribution of the three peaks and this explains the interference fringes. $q_{\theta_{L}}$ represents the quadrature of a balanced homodyne measurement. The interaction time is given by $\tau=\tau_{r} / 4$ with the revival time $\tau_{r}$ of Eq. (12), $\omega=8 m \pi / \tau_{r}\left(m \in \mathbb{N}_{0}\right)$ and the rest parameters correspond to those of Fig. 1 .

fringes in between. This is apparent from Fig. 2. These interference fringes reflect the fact that the freely evolving coherent state field state $\left|\alpha \mathrm{e}^{-i \omega \tau}\right\rangle$ has finite overlaps with the other field states $\left|\chi_{j}(\tau)\right\rangle \quad(j=0, \pm 1)$ constituting the mixed state $\hat{\rho}_{F}$. To ensure that the interval $\left(\sqrt{2}|\alpha|-\delta_{L}, \sqrt{2}|\alpha|+\delta_{L}\right)$ of the homodyne measurement does not include the interference fringes the inequality $\delta_{L}<\frac{|\alpha|}{\sqrt{2}} \sin ^{2}\left(\frac{\pi \tau}{\tau_{r}}\right)$ has to be fulfilled for the interaction time $\tau$. This inequality can be derived from the coherent state approximation by realising that the interference fringes have a Gaussian envelope and they are centered at $\sqrt{2}|\alpha| \cos ^{2}\left(\frac{\pi \tau}{\tau_{r}}\right)$ in $q_{\theta_{L}}$. In Fig. 2 we used a detection time $\tau=\tau_{r} / 4$ and $|\alpha|=7.6$ giving rise to the inequality $\delta_{L}<2.68701$, which still allows a very good probability of projecting onto the desired coherent state (compare with Eq. (B6)).

Finally, we would like to comment that in a recent study by Rodrigues et al. 26] a similar protocol was introduced for the postselective preparation of a maximally entangled state by balanced homodyne photodetection when both material qubits are prepared in the ground state. In their scheme the resulting entangled state has the inconvenience of having a time dependent relative phase. In contrast, the method presented here can produce a perfect Bell state for certain initial conditions. In the following sections we will show how to enlarge the class of initial conditions such that our method can be extended to implement quantum teleportation and entanglement swapping protocols. 


\section{A RAMSEY-TYPE PHOTONIC POSTSELECTION SCHEME}

In this section a generalization of the photonic postselection scheme of the previous section is discussed which involves a Ramsey-type matter-field interaction scenario with two cavities. Ideally it allows the probabilistic postselection of a two-qubit Bell state with unit fidelity for arbitrary initial conditions of the material qubits. This photonic postselection is achieved by projection onto a coherent state which may be achieved with the help of balanced homodyne photodetection. The success probability of this postselective Bell-state projection is determined by the initial condition of the material qubits.

Let us consider an interaction scenario as schematically depicted in Fig. 3 In a first step two qubits interact with a single mode of the radiation field inside a cavity for a time $\tau$ so that their interaction can be described by the Tavis-Cummings Hamiltonian of Eq. (1). At time $\tau$ the resulting tripartite qubit-field state is given in Eq. (5) if initially the radiation field is prepared in the coherent state $|\alpha\rangle$. In the approximation of large mean photon numbers, i.e. $\bar{n} \gg 1$, and for interaction times $\tau$ in the plateau region of Fig. 1 i.e. $\tau_{c} \ll \tau \ll \tau_{r}$, projection of the resulting tripartite qubit-field state onto the freely evolved coherent state $\left|\alpha \mathrm{e}^{-i \omega \tau}\right\rangle$ yields the twoqubit state of Eq. (14) which reduces to the maximally entangled Bell state provided the parameter $\eta(\vec{c}, \phi)$ vanishes. However, in general a vanishing value of $\eta(\vec{c}, \phi)$ can only be achieved for particular initially prepared twoqubit states.

In order to achieve a vanishing value of $\eta(\vec{c}, \phi)$ for arbitrary initial conditions of the two-qubit system a second identical interaction is enforced with a second cavity for a time $\tau$ (with $\tau_{c} \ll \tau \ll \tau_{r}$ ) after a free time evolution of the two-qubit system for a time $\tau_{f}$. The single mode of the second cavity interacting almost resonantly with the two-qubit system is initially prepared in the coherent state $\left|\alpha \mathrm{e}^{i \varphi}\right\rangle$ which differs by a phase $\varphi$ from the initially prepared coherent field state $|\alpha\rangle$ of the first cavity. The intermediate free evolution of the two-qubit system during the second step of this process is governed by the free two-qubit Hamiltonian $\hat{H}_{\mathrm{a}}=\hbar \omega_{\mathrm{a}} / 2\left(\hat{\sigma}_{A}^{z}+\hat{\sigma}_{B}^{z}\right)$. This Hamiltonian only affects the phases accumulated by the two-qubit states $|0,0\rangle$ and $|1,1\rangle$ appearing in Eq. (14). Thus, after the first photonic postselection at time $\tau+\tau_{f}$ the tripartite state involving the two material quantum systems and the mode of the second cavity is given by

$$
\left|\Psi_{1}\right\rangle=\left(\frac{c_{-}}{\sqrt{P}}\left|\Psi^{-}\right\rangle+\frac{\eta(\vec{c}, \phi) s}{\sqrt{P}}\left|\psi_{\phi-\omega_{a} \tau_{f}}\right\rangle\right)\left|\alpha \mathrm{e}^{i \tilde{\varphi}}\right\rangle
$$

with the success probability $P$ of Eq. (17) and the state $\left|\psi_{\phi-\omega_{a} \tau_{f}}\right\rangle$ defined in Eq. (16). The phase

$$
\tilde{\varphi}=\varphi-\omega\left(\tau+\tau_{f}\right)
$$

takes into account the free evolution of the coherent state in the second cavity which is assumed to be identical to the first cavity. Immediately after the threestep Ramsey-type interaction sequence, i.e. at time

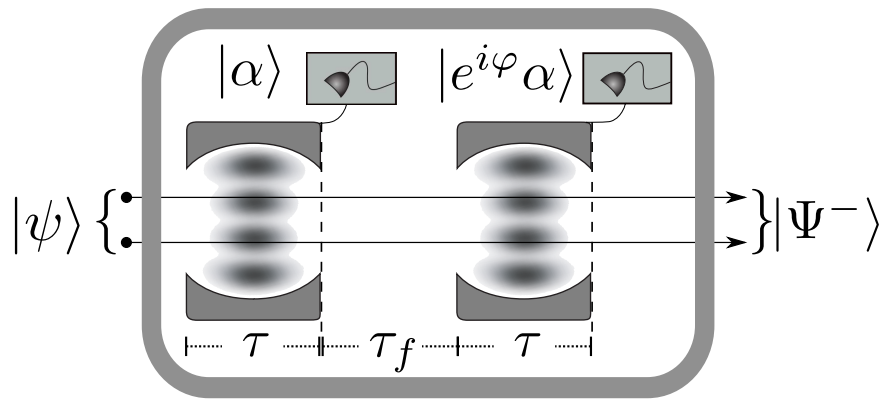

FIG. 3: Ramsey-type interaction scenario for probabilistic postselection of a two-qubit Bell state $\left|\Psi^{-}\right\rangle$: In a first step two qubits interact for a time $\tau$ with a single photonic mode inside a cavity initially prepared in the coherent state $|\alpha\rangle$. Immediately afterwards the resulting photonic state is projected onto the freely evolved coherent state $\left|\alpha \mathrm{e}^{-i \omega \tau}\right\rangle$. During the second step the two material qubits evolve freely for a time $\tau_{f}$. In the third step the two qubits interact with a second cavity initially prepared in a coherent state $\left|\mathrm{e}^{i \varphi} \alpha\right\rangle$. At time $2 \tau+\tau_{f}$ the photonic quantum state inside the second cavity is projected onto the freely evolved coherent state $\left|\alpha \mathrm{e}^{i\left(\varphi-\omega\left(2 \tau+\tau_{f}\right)\right)}\right\rangle$. Both photonic projections can be achieved by homodyne detections which are depicted as detector inside boxes. The two-qubit state resulting from this postselection process is the maximally entangled Bell state $\left|\Psi^{-}\right\rangle$. Ideally it is prepared with unit fidelity for arbitrary initial conditions of the two material qubits and with the success probability $\left|c_{-}\right|^{2}$.

$2 \tau+\tau_{f}$, the resulting two-qubit-field state is projected onto the freely evolved coherent state of the second cavity $\left|\alpha \mathrm{e}^{i(\tilde{\varphi}-\omega \tau)}\right\rangle$. This projection can be evaluated in an analogous way as in the first projected state of Eq. (14) yielding the postselected two-qubit quantum state

$$
\begin{aligned}
\left\langle\alpha \mathrm{e}^{i(\tilde{\varphi}-\omega \tau)}\left|\mathrm{e}^{-i \frac{\hat{H}^{\prime}}{\hbar} \tau}\right| \Psi_{1}\right\rangle= & \frac{c_{-}}{\sqrt{P}}\left|\Psi^{-}\right\rangle+ \\
& \eta(\vec{d}, \phi+\tilde{\varphi}) \frac{\eta(\vec{c}, \phi) s^{2}}{\sqrt{P}}\left|\psi_{\phi+\tilde{\varphi}}\right\rangle .
\end{aligned}
$$

The entries of $\vec{d}=\left(\delta / \omega_{\bar{n}}, d_{0},-d_{0}^{*}\right)$ represent the initial conditions of the state in Eq. (22) and according to the definition in Eq. (16) we get the value $d_{0}=\mathrm{e}^{-i\left(\theta-\left(\omega+\varpi_{0}\right) \tau+\phi-\omega_{\mathrm{a}} \tau_{f}\right)} / \sqrt{2}$. They have to be inserted into Eq. (10) in order to obtain explicitly

$$
\eta(\vec{d}, \phi+\tilde{\varphi})=\frac{\omega_{n}^{2}}{\Omega_{n}^{2}}\left(\frac{\delta^{2}}{\omega_{n}^{2}}+\cos \left(\tilde{\varphi}+\left(\omega+\varpi_{0}\right) \tau+\omega_{\mathrm{a}} \tau_{f}\right)\right) .
$$

The Hamiltonian $\hat{H}^{\prime}$ in Eq. (24) has the same form as Eq. (11) and we use the primed notation to distinguish the mode of the second cavity from the mode of the first cavity. The parameter $\eta(\vec{d}, \phi+\tilde{\varphi})$ can vanish if the initial phase of the second coherent state $\varphi$ is chosen in such a way that the conditions

$$
\varphi=\arccos \left(-\frac{\delta^{2}}{\omega \frac{2}{n}}\right)-\varpi_{0} \tau-\delta \tau_{f},
$$

and $\delta \leq \omega_{\bar{n}}=g \sqrt{4 \bar{n}-2}$ are fulfilled. In the case of perfect resonance $(\delta=0) \varphi$ takes the value of $\pi / 2$. If 


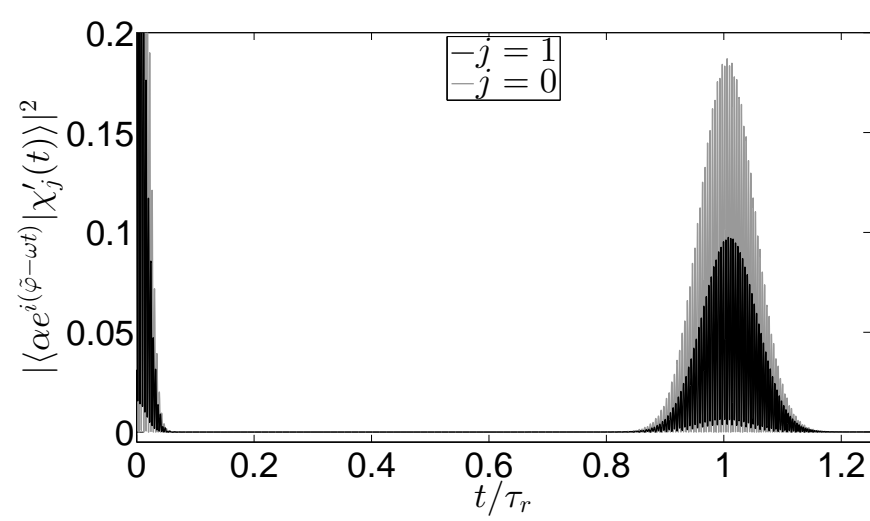

FIG. 4: Overlap between the exact photonic states $\left|\chi_{j}^{\prime}(t)\right\rangle$ of the second cavity and the coherent state $\left|\alpha \mathrm{e}^{i(\tilde{\varphi}-\omega t)}\right\rangle$ characterizing the third step of the Ramsey-type postselection scheme. The initial material state is taken from Eq. (14) for an interaction time of $\tau=\tau_{r} / 4$. The parameters $\alpha, c_{-}, c_{1}, c_{+}$, $c_{0}$, and $\delta / g$ are set to the same value as in Fig. 11 All three overlaps vanish in the plateau region.

the condition of Eq. (26) is fulfilled the projection onto the coherent state $\left|\alpha e^{i(\tilde{\varphi}-\omega \tau)}\right\rangle$ postselects the Bell state $\left|\Psi^{-}\right\rangle$and this occurs with a probability of $P^{\prime}=\left|c_{-}\right|^{2} / P$ (compare with Eq. (24)). Because both projections are independent the overall success probability of this scheme is given by

$$
P_{T}=P P^{\prime}=\left|c_{-}\right|^{2} .
$$

Both projections onto the relevant coherent states of the single-mode radiation fields can be achieved by balanced homodyne detection of the relevant photons by appropriate choices of the phases of the local oscillators. For the homodyne measurement at time $\tau$ one has to choose $\theta_{L}=\phi-\omega \tau$ and for the corresponding homodyne detection at time $2 \tau+\tau_{f}$ the phase of the local oscillator has to adjusted to the value $\theta_{L}^{\prime}=\phi+\varphi-\omega\left(2 \tau+\tau_{f}\right)$. Remarkably, this probabilistic postselective preparation of the two-qubit Bell state $\left|\Psi^{-}\right\rangle$can be achieved for arbitrary initially prepared quantum states of the two material quantum systems. Ideally this preparation can be realized with unit fidelity and with a success probability $\left|c_{-}\right|^{2}$ which depends on the initially prepared two-qubit state.

In Fig. 4the overlaps between the photonic field states $\left|\chi_{j}(t)\right\rangle$ and the freely evolved coherent state $\left|\alpha \mathrm{e}^{i(\tilde{\varphi}-\omega \tau)}\right\rangle$ are shown for the initial condition of Eq. (22). The overlaps clearly vanish for times $\tau$ in the plateau region i.e. $\tau_{c} \ll \tau \ll \tau_{r}$. The corresponding Wigner function of the field state is depicted in Fig. 5. Here, consistent with these vanishing overlaps the interference fringes between the freely evolving field state and the residual field states are not present. This demonstrates that the coherent state $\left|\alpha \mathrm{e}^{i(\tilde{\varphi}-\omega \tau)}\right\rangle$ is solely paired with the Bell state $\left|\Psi^{-}\right\rangle$which can be prepared with unit fidelity by photonic postselection.

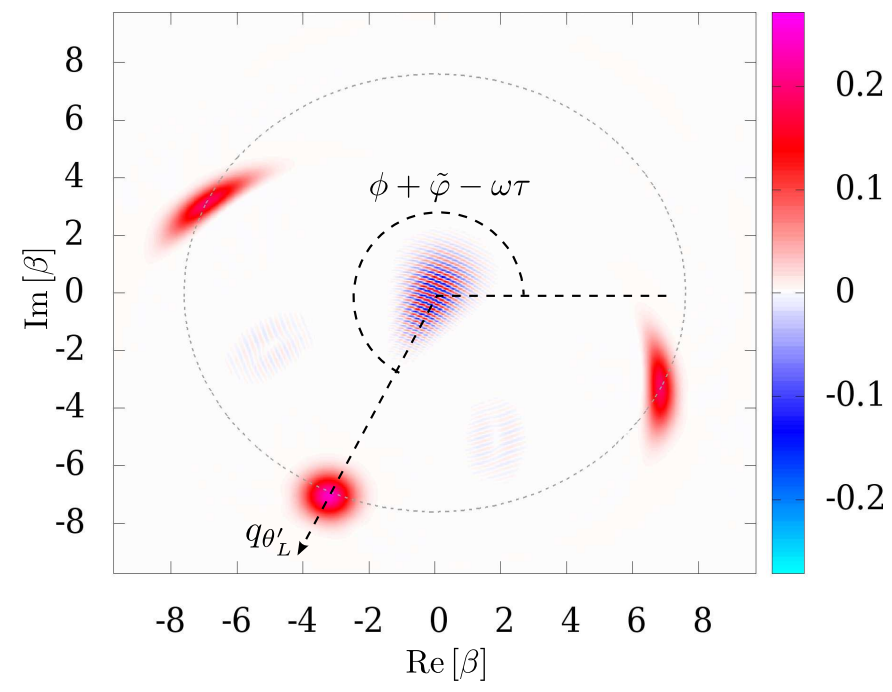

FIG. 5: Wigner phase space distribution of the photonic quantum state in the second cavity after the third step of the Ramsey-type postselection scenario: The Gaussian peak is correlated with the material state $\left|\Psi^{-}\right\rangle$. The rest of the photonic states in Eq. (5) have no contribution of the Gaussian peak. This explains the vanishing interference fringes with the other two peaks. $q_{\theta_{L}^{\prime}}$ represents the quadrature of a balanced homodyne measurement. The interaction time is given by $\tau=\tau_{r} / 4$ with the revival time $\tau_{r}$ of Eq. (12), $\omega=8 m \pi / \tau_{r}$ $\left(m \in \mathbb{N}_{0}\right)$. The other parameters correspond to those of Fig. 4

It is worth mentioning that this probabilistic preparation of the two-qubit Bell state $\left|\Psi^{-}\right\rangle$by two time-delayed homodyne measurements also works in more general situations which involve different detunings and different dipole coupling phases in both cavities, for example. In such cases one would have to add to Eq. (26) the difference between both of the dipole coupling phases, i.e. $\theta-\theta^{\prime}$, the detuning between cavities times the interaction time $\left(\omega^{\prime}-\omega\right) \tau$, and to perform the replacements $\delta^{2} / \omega_{\bar{n}}^{2} \rightarrow \delta \delta^{\prime} / \omega_{\bar{n}} \omega_{\bar{n}^{\prime}}$ and $\delta \tau_{f} \rightarrow \delta^{\prime} \tau_{f}$.

\section{DIFFERENT QUBITS}

In this section we explore the case of different coupling strengths of the qubits to the field as well as different transition frequencies. This is of interest for any experimental realization of the proposed scheme. To this end we choose to define the coupling strength of qubit $A(B)$ to the radiation field as $g_{A}=g+\varepsilon_{g},\left(g_{B}=g-\varepsilon_{g}\right)$. The transition frequency of qubit $A(B)$ is detuned from the frequency of the cavity mode as described by the equation $\delta_{A}=\delta+\varepsilon_{\delta}\left(\delta_{B}=\delta-\varepsilon_{\delta}\right)$.

In this situation the state $\left|\Psi^{-}\right\rangle|n\rangle$ is no longer an eigenstate of the Hamiltonian and therefore the time depen- 


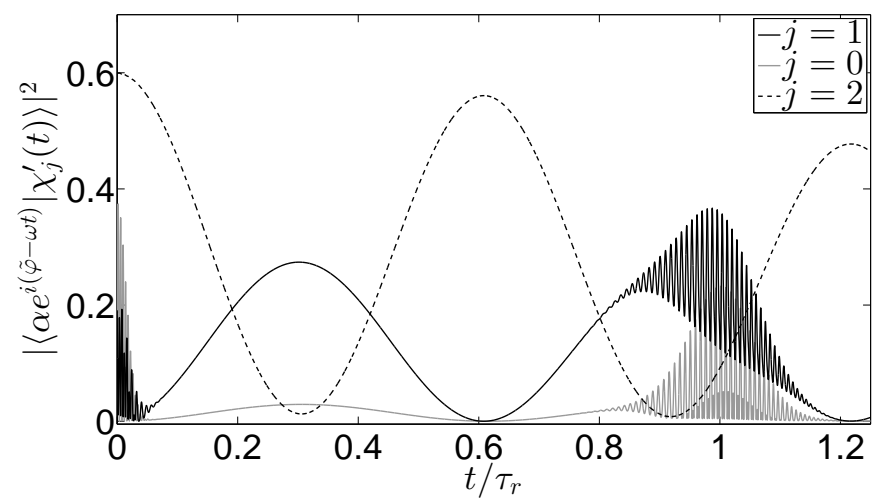

FIG. 6: Overlap between the exact photonic states $\left|\chi_{j}^{\prime}(t)\right\rangle$ of the second cavity and the coherent state $\left|\alpha \mathrm{e}^{i(\tilde{\varphi}-\omega t)}\right\rangle$ characterizing the third step of the Ramsey-type postselection scheme. The initial material state is taken from Eq. (14) for an interaction time of $\tau=\tau_{r} / 4$. The parameters $\alpha, c_{-}, c_{1}, c_{+}$, $c_{0}$, and $\delta / g$ are set to the same value as in Fig. 11 In addition we consider an asymmetry in the coupling strengths of $\varepsilon_{g} / g=0.007$ but keep equal detunings, i.e. $\varepsilon_{\delta}=0$.

dent state vector of the complete system is given by

$$
\begin{aligned}
|\Psi(t)\rangle & =\left|\Psi^{-}\right\rangle\left|\chi_{2}(t)\right\rangle \\
& +|1,1\rangle\left|\chi_{1}(t)\right\rangle+\left|\Psi^{+}\right\rangle\left|\chi_{0}(t)\right\rangle+|0,0\rangle\left|\chi_{-1}(t)\right\rangle .
\end{aligned}
$$

In contrast to Eq. (5) the photonic state $\left|\chi_{2}(t)\right\rangle$ is in general no longer a coherent state.

In Fig. 6 we present an exact numerical calculation of the overlaps of the photonic field states of the second cavity with the coherent state $\left|\alpha \mathrm{e}^{i(\tilde{\varphi}-\omega \tau)}\right\rangle$. This is the analog of Fig. 4 but with an asymmetry in the coupling strengths of $\varepsilon_{g} / g=0.007$. In addition we have included the overlap with the state $\left|\chi_{2}(t)\right\rangle$ which is not a constant. One notes the emergence of additional Rabi oscillations. In Appendix Cwe show that this Rabi frequency increases as a function of $\varepsilon_{g}$ and $\varepsilon_{\delta}$. The oscillations are also damped and undergo the typical collapse and revival phenomena.

To evaluate how unequal coupling strengths influence our scheme presented in Sec. III we evaluate the overall success probability $P_{T}$ and the fidelity $F$ of achieving the Bell state $\left|\Psi^{-}\right\rangle$. In Fig. 7 we present an exact numerical calculation for both of these quantities as a function of the difference $\varepsilon_{g}$ between couplings strengths of the qubits to the cavity mode. In this example we took equal detunings, i.e. $\varepsilon_{\delta}=0$. The fidelity displays an oscillatory behaviour and it attains values close to unity in a periodic way. The success probability also oscillates and decays as a function of $\varepsilon_{g}$. Both effects are consequences of the collapse and revival phenomena of the Rabi oscillations induced by unequal couplings.

The frequency of the Rabi oscillations increases as a function of $\varepsilon_{g}$ and the maxima of the fidelity occurs at values of $\varepsilon_{g}$ where the Rabi oscillations complete a cycle at interaction time $\tau=\tau_{r} / 4$ (compare with Eq.(12).

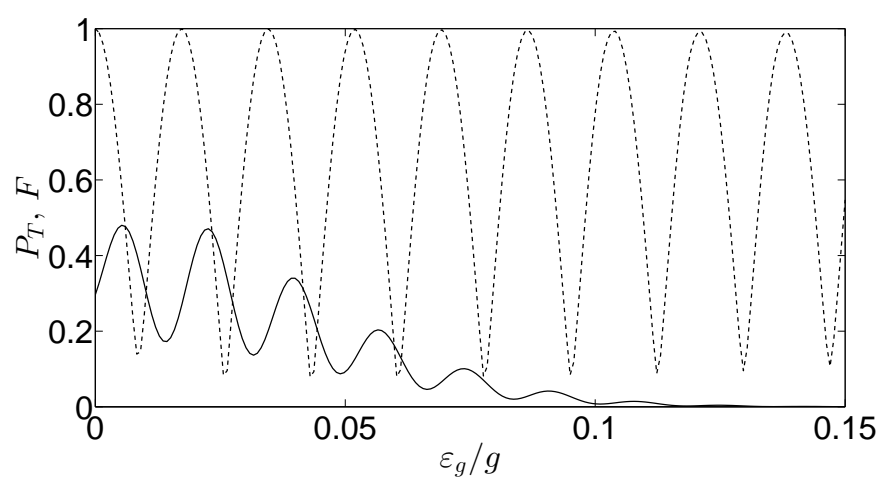

FIG. 7: Success probability $P_{T}$ (full line) and fidelity $F$ (dashed line) of achieving the state $\left|\Psi^{-}\right\rangle$as a function of the asymmetry in the coupling strengths $\varepsilon_{g}$ and using the scheme of Fig. 3. We take $\varepsilon_{\delta}=0$. The rest of the parameters and the initial conditions are the same of Fig. 1 .

We can estimate that this happens for integer multiples of $\varepsilon_{g} / g \rightarrow 4 g^{2} / \omega_{n}^{2} \approx 1 / \bar{n}$ (compare with Eq. (17). Similar behaviour of the fidelity occurs for an asymmetry in the detunings so that the cycles are completed at integer multiples of $\varepsilon_{\delta} / g \rightarrow 4 g / \delta$. We can conclude that unequal coupling strengths between the qubits to the radiation field and unequal detunings have to fulfill the requirements $\varepsilon_{g} / g \ll 1 / 2 \bar{n}$ and $\varepsilon_{\delta} / g \ll 2 g / \delta$ because it is at these values where the first minimum of the fidelity is attained. This means that the scheme is sensitive to variations of the coupling strengths but more robust with respect to small variations of the detunings. In Appendix C] we show details of the derivations of these conditions.

A possible experimental realization of the Bell projection scheme involving nowadays technology could involve flying atoms and single mode cavities. Different coupling strengths to the cavity mode can arise from the different paths on which the atoms cross the electromagnetic field mode inside the cavity. Therefore, if we consider two mirrors of a cavity facing each other along the $z$ axis, for example, a typical position dependent coupling strength can be modelled by

$$
g(x, y, z)=g_{0} \sin \left(\frac{2 \pi z}{\lambda}\right) \mathrm{e}^{-\frac{x^{2}+y^{2}}{w^{2}}}
$$

with $\lambda$ and $w$ denoting the wavelength of the cavity and the mode waist. Thereby, the spatial positions of the flying atoms are chosen so that their $x$ and $y$ coordinates are the same and they pass through the cavity at different values of $z$. In order to achieve strong coupling both atoms should be located at the antinodes of the radiation field. However, even in this case unequal couplings to the field mode may result from inaccuracies in the positions of the atomic paths. As we know that our scheme works for $\left(g_{A}-g_{B}\right) /\left(g_{A}+g_{B}\right)<1 / 2 \bar{n}$ let us address the question for which inaccuracies in the positions of the atomic paths this condition can still be fulfilled.

For this purpose let us consider the recent experiment of Ref. 27] with flying Rydberg atoms. In this experiment 
the mirrors are positioned at a distance of $2.7 \mathrm{~cm}$, the cavity is resonant at $51.1 \mathrm{GHz}$, the maximum coupling is given by $g_{0} / 2 \pi=51 \mathrm{kHz}$ and the waist is $w=6 \mathrm{~mm}$. The experienced change in the coupling strength due to the waist is well under control because the experimental study integrates the collected data over the flying time through the cavity. We can now estimate the allowed deviations $\epsilon_{z, A}, \epsilon_{z, B}$ in the positions of the atoms by

$$
\left|\frac{\sin \left(\frac{2 \pi\left(z_{A}+\epsilon_{z, A}\right)}{\lambda}\right)-\sin \left(\frac{2 \pi\left(z_{B}-\epsilon_{z, B}\right)}{\lambda}\right)}{\sin \left(\frac{2 \pi\left(z_{A}+\epsilon_{z, A}\right)}{\lambda}\right)+\sin \left(\frac{2 \pi\left(z_{B}-\epsilon_{z, B}\right)}{\lambda}\right)}\right|<1 / 2 \bar{n} .
$$

Assuming that the ideal positions $z_{A}$ and $z_{B}$ are such that $\sin \left(2 \pi z_{A} / \lambda\right)=\sin \left(2 \pi z_{B} / \lambda\right)=1$ and that the deviations $\epsilon_{z, A}, \epsilon_{z, B}$ are below $1 \mathrm{~mm}$ we obtain

$$
\left|\epsilon_{z, A}-\epsilon_{z, B}\right|<\lambda / \pi \arctan \left(\frac{1}{2 \bar{n}}\right) .
$$

Thus, if the deviations are similar for both paths, i.e. $\epsilon_{z, A}=\epsilon_{z, B}$, the above condition is always fulfilled. Otherwise, for $\bar{n} \sim 10^{2}$ the difference of these deviations must obey the relation $\left|\epsilon_{z, A}-\epsilon_{z, B}\right|<10 \mu \mathrm{m}$. Therefore, if the average number of photons $\bar{n}$ is not too large, current experiments are precise enough to realize the condition of Eq. (31).

\section{QUANTUM INFORMATION PROCESSING}

In this section it is demonstrated how the Ramseytype interaction scheme of Sec. III can be used for implementing probabilistic quantum teleportation and entanglement swapping. Thereby, the crucial feature is exploited that ideally this Ramsey-type interaction scheme allows to postselect a Bell state of two material qubits with unit fidelity for a large class of initial conditions of the two material qubits. As this postselection procedure can be implemented with the help of balanced homodyne photodetection it offers interesting perspectives for current applications in quantum information processing.

\section{A. Entanglement-assisted Teleportation}

The goal of entanglement-assisted quantum teleportation is to transfer the unknown state of a quantum system, say $A$, to another quantum system, say $C$. So, let us consider three material qubits $A, B$, and $C$ as depicted in Fig. 8 with the qubit $B$ acting as an ancilla system. Initially qubit $A$ is prepared in the unknown quantum state

$$
|\psi\rangle_{A}=a|0\rangle_{A}+b|1\rangle_{A},|a|^{2}+\left|b^{2}\right|=1 .
$$

Thus, in order to implement a photon-assisted quantum teleportation protocol let us consider the initially prepared four-partite quantum state

$$
\left|\Psi_{0}^{\mathrm{tel}}\right\rangle=|\psi\rangle_{A} \otimes\left|\Psi^{-}\right\rangle_{B C} \otimes|\alpha\rangle
$$

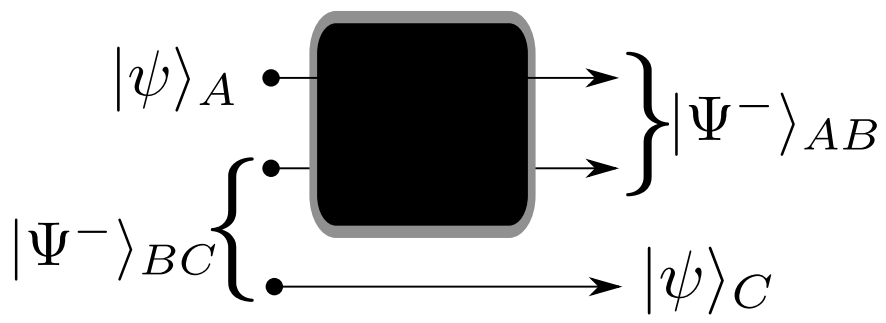

FIG. 8: A probabilistic quantum teleportation protocol based on the Ramsey-type photonic postselection scheme of the Bell state $\left|\Psi^{-}\right\rangle$as discussed in Sec. III and depicted by Fig. 3

which involves the three material qubits $A, B, C$ and the initially prepared single-mode coherent quantum state $|\alpha\rangle$ of the radiation field. This initially prepared fourpartite quantum state can be represented in the equivalent form

$$
\begin{aligned}
\left|\Psi_{0}^{\mathrm{tel}}\right\rangle & =-\frac{1}{2}\left|\Psi^{-}\right\rangle_{A B}|\psi\rangle_{C}|\alpha\rangle \\
& +\left(\frac{b}{2}\left|\Psi^{+}\right\rangle_{A B}+\frac{a}{\sqrt{2}}|00\rangle_{A B}\right)|1\rangle_{C}|\alpha\rangle \\
& -\left(\frac{b}{\sqrt{2}}|11\rangle_{A B}+\frac{a}{2}\left|\Psi^{+}\right\rangle_{A B}\right)|0\rangle_{C}|\alpha\rangle .
\end{aligned}
$$

Furthermore, let us assume that atoms $A$ and $B$ interact with the single mode of the radiation field inside a cavity so that this interaction can be described by the Hamilton operator of the Tavis-Cummings model of Eq. (11). As the Bell state $\left|\psi^{-}\right\rangle_{A B}$ is an invariant state of the Tavis-Cummings model the photonic state evolves freely as a harmonically oscillating coherent state. From our discussion in Sec. [II] it is known that after an interaction time $\tau$ with $\tau_{c} \ll \tau \ll \tau_{r}$ successful projection onto the photonic state $\left|\alpha e^{-i \omega \tau}\right\rangle$ results in the unnormalized tripartite material quantum state (see Eq. (14))

$$
\begin{aligned}
\left|\psi^{\text {tel }}\right\rangle & =\left\langle\alpha \mathrm{e}^{-i \omega t} \mid \Psi^{\mathrm{tel}}(\tau)\right\rangle=-\frac{1}{2}\left|\Psi^{-}\right\rangle_{A B}|\psi\rangle_{C}+ \\
& +s\left|\psi_{\phi}\right\rangle_{A B}\left(\eta\left(\vec{d}_{1}, \phi\right)|1\rangle_{C}+\eta\left(\vec{d}_{0}, \phi\right)|0\rangle_{C}\right) .
\end{aligned}
$$

Thereby, the state $\left|\psi_{\phi}\right\rangle_{A B}$ is given by Eqs. (14), (15), (16) and the amplitudes in Eq. (35) are defined by the initial conditions encoded in the vectors $\overrightarrow{d_{1}}=(b / 2, a / \sqrt{2}, 0)$ and $\overrightarrow{d_{0}}=(-a / 2,0,-b / \sqrt{2})$ according to the definition of $\eta(\vec{c}, \phi)$ in Eq. (10).

This projection onto the state (35) takes place with probability

$$
P^{\mathrm{tel}}=\frac{1}{4}+|s|^{2}\left(\left|\eta\left(\vec{d}_{0}, \phi\right)\right|^{2}+\left|\eta\left(\vec{d}_{1}, \phi\right)\right|^{2}\right)\left(1+\frac{\delta^{2}}{\omega_{n}^{2}}\right) .
$$

Now, let us assume that subsequently the quantum systems $A$ and $B$ interact for a time $\tau$ with a second cavity prepared in the single-mode coherent state $\left|\alpha e^{i \varphi}\right\rangle$. If the relative phase $\varphi$ fulfills the condition of Eq. (26) a second projection onto the freely evolved coherent state $\left|\alpha e^{i(\tilde{\varphi}-\omega \tau)}\right\rangle$ results in the teleported quantum state

$$
\left|\Phi^{\mathrm{tel}}\right\rangle=\mathrm{e}^{i \pi}\left|\Psi^{-}\right\rangle_{A B} \otimes|\psi\rangle_{C} .
$$


This second photonic projection takes place with probability $P^{\text {tel }}=1 /\left(4 P^{\text {tel }}\right)$ so that the overall success probability of this entanglement-assisted quantum teleportation protocol is independent of the initial conditions of the state to be teleported and is given by

$$
P_{T}^{\mathrm{tel}}=0.25
$$

\section{B. Entanglement swapping}

A major aim of entanglement swapping is to produce entanglement between two distant quantum systems, say $C$ and $D$, with the help of two uncorrelated pairs of entangled quantum systems, say $A D$ and $B C$. Let us consider four material qubits $A, B, C$, and $D$ as depicted in Fig. 9. Initially, the qubit pairs $B C$ and $A D$ are prepared in maximally entangled Bell states and an ancillary photonic field mode is prepared in a coherent state $|\alpha\rangle$ so that the five-partite initially prepared quantum state is given by

$$
\left|\Psi_{0}^{\mathrm{sw}}\right\rangle=\left|\Psi^{ \pm}\right\rangle_{D A}\left|\Psi^{-}\right\rangle_{B C}|\alpha\rangle .
$$

This initial state can be represented in the equivalent form

$$
\begin{aligned}
\left|\Psi_{0}^{\mathrm{sw}}\right\rangle & =-\frac{1}{2}\left|\Psi^{-}\right\rangle_{A B}\left|\Psi^{ \pm}\right\rangle_{D C}|\alpha\rangle-\frac{1}{2}|1,1\rangle_{A B}|0,0\rangle_{D C}|\alpha\rangle \\
& +\frac{1}{2}\left|\Psi^{+}\right\rangle_{A B}\left|\Psi^{\mp}\right\rangle_{D C}|\alpha\rangle \pm \frac{1}{2}|0,0\rangle_{A B}|1,1\rangle_{D C}|\alpha\rangle .
\end{aligned}
$$

A Bell projection on qubits $A$ and $B$ is capable of swapping entanglement to qubits $C$ and $D$. For this purpose qubits $A$ and $B$ interact with the ancillary photonic field mode inside a cavity for a time $\tau$ with $\tau_{c} \ll \tau \ll \tau_{r}$. If this interaction can be described by the Tavis-Cummings Hamiltonian of Eq. (1) we can take advantage from the fact that the two-qubit Bell state $\left|\Psi^{-}\right\rangle_{A B}$ is an invariant quantum state under the Hamiltonian of Eq.(11) so that the photonic field state it is correlated with according to Eq. (40) evolves freely as an oscillating coherent state. Thus, projection of the five-partite quantum state $|\Psi(\tau)\rangle$ onto the coherent state $\left|\alpha e^{-i \omega \tau}\right\rangle$ yields the unnormalized four-partite qubit state (see Eq. (14) )

$$
\begin{aligned}
&\left|\psi^{\mathrm{sw}}\right\rangle=\langle\left\langle\mathrm{e}^{-i \omega \tau} \mid \Psi^{\mathrm{sw}}(\tau)\right\rangle=-\frac{1}{2}\left|\Psi^{-}\right\rangle_{A B}\left|\Psi^{ \pm}\right\rangle_{D C}+ \\
& s\left|\psi_{\phi}\right\rangle_{A B}( \eta\left(\vec{d}_{00}, \phi\right)|0,0\rangle_{D C}+\eta\left(\vec{d}_{+}, \phi\right)\left|\Psi^{\mp}\right\rangle_{D C}+ \\
&\left.\eta\left(\vec{d}_{11}, \phi\right)|1,1\rangle_{D C}\right)
\end{aligned}
$$

with the initial conditions of Eq. (40) represented by the vectors $\vec{d}_{00}=(0,0,-1 / 2), \vec{d}_{+}=(1 / 2,0,0)$ and $\vec{d}_{11}=(0, \pm 1 / 2,0)$. These initial conditions have to be substituted into the definition of $\eta(\vec{c}, \phi)$ in Eq. (10). The success probability of this photonic projection is given by

$$
\begin{aligned}
P^{\mathrm{sw}} & =\frac{1}{4}+|s|^{2}\left(1+\frac{\delta^{2}}{\omega_{\bar{n}}^{2}}\right) \times \\
& \left(\left|\eta\left(\vec{d}_{+}, \phi\right)\right|^{2}+\left|\eta\left(\vec{d}_{00}, \phi\right)\right|^{2}+\left|\eta\left({\overrightarrow{d_{11}}}_{1} \phi\right)\right|^{2}\right) .
\end{aligned}
$$

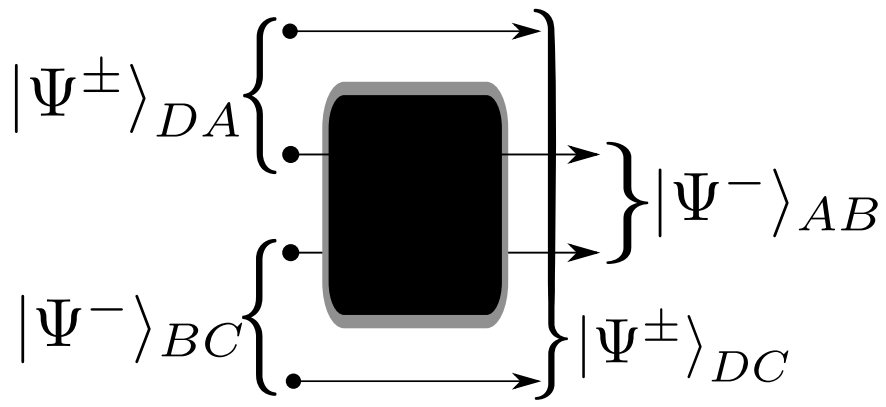

FIG. 9: A probabilistic entanglement swapping protocol based on the Ramsey-type photonic postselection scheme of the Bell state $\left|\Psi^{-}\right\rangle$as discussed in Sec. III and depicted by Fig. 3

In order to achieve projection onto the Bell state $\left|\Psi^{-}\right\rangle_{A B}$ with unit fidelity, qubits $A$ and $B$ interact with a second single mode of the radiation field inside a second cavity for a time $\tau$. Thereby, the radiation field is prepared in a coherent state $\left|\alpha e^{i \varphi}\right\rangle$ so that condition (26) is fulfilled. According to our discussion in section III after the projection onto the second coherent state $\left|\alpha e^{i(\tilde{\varphi}-\omega \tau)}\right\rangle$ the final four-partite qubit state is given by

$$
\left|\Phi^{\mathrm{sw}}\right\rangle=\mathrm{e}^{i \pi}\left|\Psi^{-}\right\rangle_{A B}\left|\Psi^{ \pm}\right\rangle_{D C}
$$

This second photonic projection is achieved with a success probability of $1 /\left(4 P^{\mathrm{sw}}\right)$. Multiplying the probabilities of both photonic projections yields the overall success probability of this probabilistic entanglement swapping procedure, namely

$$
P_{T}^{\mathrm{sw}}=0.25
$$

Let us finally address the question to which extent the entanglement swapping procedure discussed here may offer interesting perspectives for current experimental activities in realizing a quantum repeater. The experiments of Gleyzes et al. 27] have demonstrated that controlled interaction between Rydberg atoms crossing several cavities and interacting with single modes of the radiation field prepared inside these cavities is possible. Thus, the entanglement swapping protocol discussed here may be integrated in a hybrid quantum repeater setup as proposed by van Loock et. al. 20] or in a setup based on almost resonant matter-field interaction [28], for example, in the following way. In a first step entanglement is generated between neighboring stations by passing material qubits through different cavities. Due to lossy transmission channels between the stations entanglement purification 29] may be performed. In a second step the previously discussed entanglement swapping procedure is applied at each station. Even if the qubits $A$ and $B$ are destroyed after the entanglement swapping procedure of Fig. 9 qubits $D$ and $C$ are still prepared in a Bell state $\left|\Psi^{ \pm}\right\rangle_{D C}$. Problems arising from the fact that radiatively long lived stable electronic levels should be used as material qubits may be resolved with the help of appropriately 
applied $\pi$-pulses. They transform radiatively long lived electronic states to higher electronic levels which can be excited almost resonantly by photons easily. Furthermore, recent experiments indicate that also the condition of negligible spontaneous photon emission into other modes of the radiation field during the interaction between the qubits and the almost resonantly coupled cavity modes can be fulfilled. Although the direct experimental investigation of the two-qubit Tavis-Cummings model by Casabone et al. [30], for example, performed on trapped ${ }^{40} \mathrm{Ca}^{+}$ions reports a ratio between the vacuum Rabi frequency $g$ and the spontaneous decay rate $\Gamma$ of the qubits as small as $g / \Gamma=0.68$, the experiment of Colombe et al. 31] reports significantly higher ratios as large as $g / \Gamma=71.66$. Thus, the experimental realization of the dynamical regime of negligible spontaneous photon emission into other modes of the radiation field is within reach of nowadays experimental possibilities.

\section{CONCLUSIONS}

We have discussed a quantum electrodynamical implementation of a probabilistic Bell measurement capable of projecting an arbitrary initial state of two material qubits perfectly onto a Bell state with success probability given by the initial probability weight of this Bell state. It has been demonstrated how this Bell measurement can be used as a building block for implementations of entanglement-assisted teleportation and entanglement swapping protocols both of which can be achieved with almost unit fidelity and $25 \%$ success probability. This Bell measurement is performed by entangling the two material qubits to be measured with single modes of the radiation field in a Ramsey-type interaction sequence and postselecting the resulting photon fields with the help of balanced homodyne photodetection. Within the dipoleand rotating wave approximation the almost resonant quantum electrodynamical matter-photon interaction involved in this Bell measurement can be described by the two-qubit Tavis-Cummings model. The protocols presented take advantage of a characteristic feature of this particular interaction model, namely the existence of an invariant two-qubit Bell state which does not couple to the photons. Therefore, if initially the ancillary photon fields are prepared in coherent states this invariant Bell state will always remain correlated with these coherent states which evolve freely despite the presence of the quantum electrodynamical matter-photon coupling. If the interaction times of the Ramsey-type interaction sequence and the initial phases of the coherent photon states are chosen appropriately ideally these coherent states can be distinguished perfectly from the residual photon states which are correlated with the other components of the material two-qubit quantum state. This offers the possibility to postselect these coherent components of the photon state by balanced homodyne photodetection thus preparing a perfect material two-qubit
Bell state with unit fidelity independently of the twoqubit state which has been prepared before the interaction with the radiation field. The properly chosen interaction times and phases of the coherent photon states involved in this Ramsey-type interaction sequence exploit characteristic dynamical properties of the collapse and revival phenomena of the Tavis-Cummings model and ensure that this postselective unit-fidelity Bell state projection can be achieved. It is this latter property which enables the use of this probabilistic Bell measurement as a basic building block for probabilistic entanglementassisted quantum teleportation. Furthermore, this probabilistic Bell measurement may also be used for implementing entanglement swapping and may thus be of particular interest for current experimental efforts aiming at the realization of hybrid quantum repeaters.

In view of significant recent progress in quantum state engineering and in the distribution of remote entanglement the postselective Bell measurement, the quantum teleportation and entanglement swapping protocols discussed here may offer interesting perspectives for future applications. Possible applications may not only include quantum optical implementations of hybrid quantum repeaters and quantum communication networks but also condensed-matter implementations of qubits which are almost resonantly coupled to coherent states of microwave fields.

\section{Acknowledgments}

This work is supported by the BMBF project Q.com.

\section{Appendix A: Time evolution of the almost resonant two-qubit Tavis-Cummings model}

In this section the time evolution of the two-qubit Tavis-Cummings model is discussed. Let us consider the situation of an almost resonant coupling between the qubits and the single mode of the cavity field. For simplicity we omit the labels of the qubits while taking the convention of keeping the order $A, B$, i.e. $|1\rangle_{A}|0\rangle_{B}=$ $|1,0\rangle$. It is apparent that the state $\left|\Psi^{-}\right\rangle|n-1\rangle$ is an eigenstate of the Hamiltonian in Eq. (11). Furthermore, the number of excitations of the two-qubit-field system $\hat{a}^{\dagger} \hat{a}+\frac{1}{2}\left(\hat{\sigma}_{A}^{z}+\hat{\sigma}_{B}^{z}\right)$ is a constant of motion of the Hamiltonian (1) of the Tavis-Cummings model. This number of excitations is diagonal in the basis

$$
\begin{aligned}
& \left\{\left|\Psi^{-}\right\rangle|n\rangle\right\}_{n=0}^{\infty} \oplus\{|0,0\rangle|0\rangle\} \oplus \\
& \left\{\left|\Psi^{+}\right\rangle|0\rangle,|0,0\rangle|1\rangle\right\} \oplus \\
& \left\{|1,1\rangle|n-2\rangle,\left|\Psi^{+}\right\rangle|n-1\rangle,|0,0\rangle|n\rangle\right\}_{n=2}^{\infty},
\end{aligned}
$$

and has a 3-fold degenerate spectrum for any fixed value of $n>1$ (2- and 1-fold degeneracy for $n=1,0$, respectively). Because the Hamilton of Eq. (1) commutes with 
the number of excitation, it follows that it can be diagonalized in blocks given by

$$
\begin{aligned}
& H^{(0)}=-\hbar \delta \\
& H^{(1)}=\hbar\left(\begin{array}{cc}
0 & g \mathrm{e}^{i \theta} \sqrt{2} \\
g \mathrm{e}^{-i \theta} \sqrt{2} & -\delta
\end{array}\right), \\
& H^{(n \geq 2)}= \\
& \hbar\left(\begin{array}{ccc}
\delta+\omega(n-1) & g \mathrm{e}^{i \theta} \sqrt{2(n-1)} & 0 \\
g \mathrm{e}^{-i \theta} \sqrt{2(n-1)} & \omega(n-1) & g \mathrm{e}^{i \theta} \sqrt{2 n} \\
0 & g \mathrm{e}^{-i \theta} \sqrt{2 n} & \omega(n-1)-\delta
\end{array}\right) .
\end{aligned}
$$

We observe that the state $|0,0\rangle|0\rangle$ is an eigenstate of the system with eigenvalue $E^{(0)}=-\hbar \delta$. For the second block we find that there are two eigenvalues given by $E_{j}^{(1)}=$ $\hbar \frac{j}{2}\left(\sqrt{8 g^{2}+\delta^{2}}\right)$ with $j=-1,1$. The solution of the eigenvalue problem for $n \geq 2$ involves the diagonalization of the $3 \times 3$ matrices $H^{(n \geq 2)}$ of Eq. (A2) and leads to a characteristic polynomial of third order. Its general solutions are lengthly [32] and not of much interest for our purposes. For large photon numbers the approximate eigenvalues of the system can be obtained with the help of perturbation theory. Choosing $\epsilon_{n}=1 / \sqrt{8 n-4}$ as an expansion parameter for each block we obtain the result

$$
\begin{aligned}
& H^{(n)}=H_{0}^{(n)}+\epsilon_{n} H_{1}^{(n)}+\ldots \\
& \frac{H_{0}^{(n)}}{\hbar}=\left(\begin{array}{ccc}
\omega(n-1)+\delta & g \mathrm{e}^{i \theta} \sqrt{2 n-1} & 0 \\
g \mathrm{e}^{-i \theta} \sqrt{2 n-1} & \omega(n-1) & g \mathrm{e}^{i \theta} \sqrt{2 n-1} \\
0 & g \mathrm{e}^{-i \theta} \sqrt{2 n-1} & \omega(n-1)-\delta
\end{array}\right), \\
& H_{1}^{(n)}=\hbar\left(\begin{array}{ccc}
0 & -g \mathrm{e}^{i \theta} & 0 \\
-g \mathrm{e}^{-i \theta} & 0 & g \mathrm{e}^{i \theta} \\
0 & g \mathrm{e}^{-i \theta} & 0
\end{array}\right) .
\end{aligned}
$$

The eigenvalues of the zeroth order blocks are $\hbar \omega(n-1)$ and $\hbar \omega(n-1) \pm \hbar \Omega_{n}$ with

$$
\Omega_{n}=\sqrt{(4 n-2) g^{2}+\delta^{2}} .
$$

The eigenvectors of these zeroth order blocks are given by the columns of the unitary matrix

$$
U_{0}^{(n)}=\left(\begin{array}{ccc}
-\frac{\omega_{n} \mathrm{e}^{i \theta}}{\sqrt{2} \Omega_{n}} & \frac{\delta-\Omega_{n}}{2 \Omega_{n}} \mathrm{e}^{i \theta} & \frac{\delta+\Omega_{n}}{2 \Omega_{n}} \mathrm{e}^{i \theta} \\
\frac{\delta}{\Omega_{n}} & \frac{\omega_{n}}{\sqrt{2} \Omega_{n}} & \frac{\omega_{n}}{\sqrt{2} \Omega_{n}} \\
\frac{\omega_{n} \mathrm{e}^{-i \theta}}{\sqrt{2} \Omega_{n}} & \frac{\omega_{n}^{2} \mathrm{e}^{-2 \theta}}{2 \Omega_{n}\left(\delta-\Omega_{n}\right)} & \frac{\omega_{n}^{2} \mathrm{e}^{-i \theta}}{2 \Omega_{n}\left(\Omega_{n}+\delta\right)}
\end{array}\right) .
$$

The corrections of first order in $\epsilon_{n}$ of the eigenvalues are given by the diagonal elements of the matrices $\epsilon_{n} U_{0}^{(n)^{\dagger}} H_{1}^{(n)} U_{0}^{(n)}$. Using these corrections up to first order in $\epsilon_{n}$ the eigenvalues are given by

$$
E_{j}^{(n)}=\hbar\left(\omega(n-1)+j \Omega_{n}+\frac{(-1)^{j}(2)^{1-|j|} g^{2} \delta}{\Omega_{n}^{2}}\right)
$$

with $j=-1,0,1$. It should be mentioned that these results are valid for arbitrary detunings $\delta$ from resonance.
Let us now determine the time evolution of the twoqubit-field quantum state with the initial condition

$$
\begin{aligned}
\left|\Psi_{0}\right\rangle & =\left(c_{-}\left|\Psi^{-}\right\rangle+c_{1}|1,1\rangle+c_{+}\left|\Psi^{+}\right\rangle+c_{0}|0,0\rangle\right) \otimes \\
& \otimes\left(\sum_{n=0}^{\infty} p_{n}|n\rangle\right)
\end{aligned}
$$

fulfilling the normalization condition

$$
\left(\left|c_{-}\right|^{2}+\left|c_{+}\right|^{2}+\left|c_{0}\right|^{2}+\left|c_{1}\right|^{2}\right)\left(\sum_{n=0}^{\infty}\left|p_{n}\right|^{2}\right)=1 .
$$

Using the zeroth order eigenvectors in $\epsilon_{n}$ and the corresponding first order eigenvalues the time evolution is approximately given by

$$
\begin{aligned}
|\Psi(t)\rangle & =c_{-}\left|\Psi^{-}\right\rangle \otimes\left(\sum_{n=0}^{\infty} p_{n} e^{-i n \omega t}|n\rangle\right)+ \\
& +|1,1\rangle\left|\chi_{1}(t)\right\rangle+\left|\Psi^{+}\right\rangle\left|\chi_{0}(t)\right\rangle+|0,0\rangle\left|\chi_{-1}(t)\right\rangle
\end{aligned}
$$

with

$$
\begin{aligned}
& \left|\chi_{1}(t)\right\rangle=\sum_{n=2}^{\infty} \sum_{k=-1}^{1} \eta_{1, k}^{(n, t)} \mathrm{e}^{i \theta}|n-2\rangle, \\
& \left|\chi_{0}(t)\right\rangle=\sum_{n=1}^{\infty} \sum_{k=-1}^{1} \eta_{0, k}^{(n, t)}|n-1\rangle, \\
& \left|\chi_{-1}(t)\right\rangle=\sum_{n=1}^{\infty} \sum_{k=-1}^{1} \eta_{-1, k}^{(n, t)} \mathrm{e}^{-i \theta}|n\rangle+\mathrm{e}^{i \delta t} c_{0} p_{0}|0\rangle
\end{aligned}
$$

and with the definitions

$$
\begin{aligned}
& \eta_{j, \pm 1}^{(n, t)}=\frac{\omega_{n}^{2}}{\sqrt{2^{\mid j}} \Omega_{n}^{2}}\left(\frac{\delta \pm \Omega_{n}}{\omega_{n}}\right)^{j}\left(\frac{c_{+} p_{n-1}}{2}+\frac{\omega_{n} c_{0} \mathrm{e}^{i \theta} p_{n}}{2 \sqrt{2}\left(\delta \pm \Omega_{n}\right)}+\right. \\
& \left.+\frac{\left(\delta \pm \Omega_{n}\right) c_{1} \mathrm{e}^{-i \theta} p_{n-2}}{2 \sqrt{2} \omega_{n}}\right) \mathrm{e}^{-i E_{ \pm 1}^{(n)} t / \hbar}, \\
& \eta_{j, 0}^{(n, t)}=\left(\frac{\delta}{\omega}\right)^{\delta_{j, 0}}(-1)^{\delta_{j, 1}} \frac{\omega_{n}^{2}}{\sqrt{2^{|j|} \mid} \Omega_{n}^{2}}\left(\frac{\delta c_{+} p_{n-1}}{\omega_{n}}+\right. \\
& \left.+\frac{c_{0} \mathrm{e}^{i \theta} p_{n}-c_{1} \mathrm{e}^{-i \theta} p_{n-2}}{\sqrt{2}}\right) \mathrm{e}^{-i E_{0}^{(n)} t / \hbar} .
\end{aligned}
$$

In the case of an initially prepared coherent photon state the probability amplitudes are given by

$$
p_{n}=\sqrt{\frac{\bar{n}^{n}}{n !}} \mathrm{e}^{-\frac{\bar{n}}{2}+i \phi} .
$$

In order to obtain an expansion in terms of coherent states one may perform a Taylor expansion of the eigenfrequencies up to first order in $n$ around the mean photon number $\bar{n} \gg 1$. Thus, the eigenvalues take the form of Eq. (6) with the definitions of Eq. (7). In the limit $\bar{n} \gg$ $\sqrt{\bar{n}}$ summations over photon numbers $n$ may be restricted approximately to intervals $n \in[\bar{n}-4 \sqrt{\bar{n}}, \bar{n}+4 \sqrt{\bar{n}}]$. Thus, the probability amplitudes of the single-mode radiation field simplify to

$$
p_{n}=\sqrt{\frac{\bar{n}}{n}} \mathrm{e}^{i \phi} p_{n-1} \approx \mathrm{e}^{i \phi} p_{n-1}
$$


and the functions of Eq. (A9) can be approximated by

$$
\eta_{j, k}^{(n, t)} \approx \eta_{j, k} \mathrm{e}^{i\left[j\left(\phi-\left(\omega+\varpi_{k}\right)(n-1) t\right)-\Delta_{k} t\right]} p_{n-j-1} .
$$

Substituting these approximations into Eq. (A8) we arrive at the result of Eq. (9). Thereby, the interaction times are restricted by the condition of Eq. (8).

\section{Perfect resonance $\delta=0$}

In the resonant case the exact solutions have remarkably compact form. For real-valued dipole couplings, for example, i.e. $\theta=0$, the blocks of the Hamiltonian $H^{(n)}$ can be diagonalized by the transformations

$$
\begin{aligned}
U^{(1)} & =\frac{1}{\sqrt{2}}\left(\begin{array}{cc}
1 & 1 \\
-1 & 1
\end{array}\right), \\
U^{(n \geq 2)} & =\frac{1}{\sqrt{4 n-2}}\left(\begin{array}{ccc}
\sqrt{2 n} & \sqrt{n-1} & \sqrt{n-1} \\
0 & -\sqrt{2 n-1} & \sqrt{2 n-1} \\
\sqrt{2 n-2} & \sqrt{n} & \sqrt{n}
\end{array}\right) .
\end{aligned}
$$

Thereby, $U^{(n)^{\dagger}} H^{(n)} U^{(n)}$ is the diagonal matrix of eigenvalues. These eigenvalues are given by $E_{j}^{n}=\hbar(\omega(n-$ 1) $+j g \sqrt{4 n-2})$ with $j=-1,0,1(j=1,-1)$ for $n>1$ $(n=1)$.

The resulting time evolution of an initial state of the form of Eq. (2) has the form of the state vector in equation (5) with the field states given by

$$
\begin{aligned}
\left|\chi_{1}(t)\right\rangle & =\sum_{n=2}^{\infty} \mathrm{e}^{i \theta} \frac{\sqrt{n-1}\left(\xi_{n, t}^{-}-\xi_{n, t}^{+}\right)-\sqrt{n} \xi_{n}}{\sqrt{2 n-1}}|n-2\rangle, \\
\left|\chi_{0}(t)\right\rangle & =\sum_{n=1}^{\infty}\left(\xi_{n, t}^{-}+\xi_{n, t}^{+}\right)|n-1\rangle, \\
\left|\chi_{-1}(t)\right\rangle & =c_{0} p_{0}|0\rangle+\sum_{n=1}^{\infty} \mathrm{e}^{-i \theta} \frac{\sqrt{n}\left(\xi_{n, t}^{-}-\xi_{n, t}^{+}\right)+\sqrt{n-1} \xi_{n}}{\sqrt{2 n-1}}|n\rangle
\end{aligned}
$$

with

$$
\begin{aligned}
\xi_{n, t}^{ \pm} & =\frac{\mathrm{e}^{ \pm i \omega_{n} t}}{2}\left(c_{+} p_{n-1} \mp \frac{\sqrt{n} c_{0} \mathrm{e}^{i \theta} p_{n}+\sqrt{n-1} c_{1} \mathrm{e}^{-i \theta} p_{n-2}}{\sqrt{2 n-1}}\right), \\
\xi_{n} & =\frac{\sqrt{n-1} c_{0} \mathrm{e}^{i \theta} p_{n}-\sqrt{n} c_{1} \mathrm{e}^{-i \theta} p_{n-2}}{\sqrt{2 n-1}},
\end{aligned}
$$

and with $p_{n}$ denoting the photon number probability amplitudes which are given by Eq. (A10) in the case of a coherent state.

\section{Appendix B: Homodyne photodetection as a projective measurement}

For the sake of completeness in this appendix we summarize basic facts about balanced homodyne photodetection measurements which are relevant for our discussion in Sec. III and which have been reviewed in detail by Lvovsky and Raymer [23], for example. In particular, we summarize the approximations which allow one to describe a homodyne photodetection measurement by a projective von Neumann measurement as in Eq. (18).

In a typical balanced homodyne photodetection experiment a single mode of the radiation field to be measured is superposed with the single mode of a local oscillator with the help of a $50 \%$ beam splitter. Ideally this process can be described by the canonical transformation

$$
\left(\begin{array}{l}
\hat{c}_{2} \\
\hat{c}_{1}
\end{array}\right)=\frac{1}{\sqrt{2}}\left(\begin{array}{cc}
1 & 1 \\
-1 & 1
\end{array}\right)\left(\begin{array}{l}
\hat{a}_{S} \\
\hat{a}_{L}
\end{array}\right)
$$

with $\hat{a}_{S}$ denoting the destruction operator of the field mode to be measured and $\hat{a}_{L}$ the mode of the local oscillator. The destruction operators of the field modes emerging from the beam splitter are denoted by $\hat{c}_{1}$ and $\hat{c}_{2}$. With the help of two photodetectors one measures the resulting difference of photon numbers which is described by the hermitian operator $\hat{n}_{-}=\hat{c}_{1}^{\dagger} \hat{c}_{1}-\hat{c}_{2}^{\dagger} \hat{c}_{2}$. According to the photodetection theory of Kelley and Kleiner [33] the probability of detecting $n_{-}=n_{1}-n_{2}$ photons is given by

$$
\begin{aligned}
& P\left(n_{-}\right)=\operatorname{Tr}\left\{\hat{\rho}_{L} \otimes \hat{\rho}_{S}\right. \\
& \left.: e^{-\xi\left(\hat{n}_{1}+\hat{n}_{2}\right)}\left(\frac{\hat{n}_{1}}{\hat{n}_{2}}\right)^{n_{-} / 2} I_{\left|n_{-}\right|}\left(2 \xi \sqrt{\hat{n}_{1} \hat{n}_{2}}\right):\right\}
\end{aligned}
$$

with the mean photon numbers

$$
n_{j}=\operatorname{Tr}\left\{\hat{\rho}_{L} \otimes{\hat{\rho_{S}}}_{\hat{c}_{j}^{\dagger}}^{\dagger} \hat{c}_{j}\right\}, \quad(j=1,2) .
$$

Thereby, it is assumed that the two field modes described by the destruction operators $\hat{a}_{L}$ and $\hat{a}_{S}$ are statistically independent and are initially prepared in the separable quantum states $\hat{\rho}_{L}$ and $\hat{\rho}_{S}$. The quantity $0 \leq \xi \leq 1$ denotes the quantum efficiency of the photodetection process and $I_{n}$ denotes the modified Bessel function of integer order $n$. Furthermore, normal ordering of an operator $\hat{O}$ with respect to the destruction and creation operators $\hat{a}_{j}$ and $\hat{a}_{j}^{\dagger}(j \in\{L, S\})$ is denoted by : $\hat{O}$ :.

If the magnitude of the difference of the photon numbers $n_{-}$is much less than the mean photon numbers of both modes emerging from the beam splitter, i.e. $\left|n_{-}\right| \ll n_{1}, n_{2}$, and in addition the local oscillator is initially prepared in a coherent state ||$\alpha_{L}\left|e^{i \theta_{L}}\right\rangle$ with $\left|\alpha_{L}\right|^{2} \gg \operatorname{Tr}\left\{\hat{\rho}_{S} \hat{a}_{S}^{\dagger} \hat{a}_{S}\right\}, 1$ this photodetection probability simplifies to the expression

$$
\begin{aligned}
& P_{\theta_{L}}\left(n_{-}\right)=\operatorname{Tr}\left\{\hat{\rho}_{S}\right. \\
& \left.: \frac{1}{\sqrt{2 \pi \xi\left|\alpha_{L}\right|^{2}}} \mathrm{e}^{-\frac{\left(n_{-}-\xi\left|\alpha_{L}\right|\left(\hat{a}_{S} e^{-i \theta_{L}}+\hat{a}_{S}^{\dagger} e^{i \theta_{L}}\right)\right)^{2}}{2 \xi\left|\alpha_{L}\right|^{2}}}:\right\} .
\end{aligned}
$$

Therefore, if the balanced homodyne detection measurement is ideal, i.e. $\xi \rightarrow 1$, the resulting probability 
of detecting a difference photon number $n_{-}$simplifies to the expression

$$
P_{\theta_{L}}\left(q_{\theta_{L}}\right)=\int d^{2} \beta W\left(\beta, \beta^{*}\right) \delta\left(q_{\theta_{L}}-\frac{\left(\beta e^{-i \theta_{L}}+\beta^{*} e^{i \theta_{L}}\right)}{\sqrt{2}}\right)
$$

with $q_{\theta_{L}}=n_{-} / \sqrt{2}\left|\alpha_{L}\right|$ and with $W\left(\beta, \beta^{*}\right)$ denoting the Wigner function of the photonic quantum state $\hat{\rho}_{S}$ as given by Eq. (21) and with $\delta(x)$ denoting the Dirac delta distribution. Using the quadrature eigenstates of Eq. (19) the probability distribution of Eq.(B4) can be rewritten in the equivalent form of Eq.(18). This form demonstrates explicitly that in this limit balanced homodyne detection of photons can be described by a projective von Neumann measurement. According to Eq.(B2), however, in general balanced homodyne detection has to be described by a positive operator valued measure.

For a coherent state $|\alpha\rangle$, for example, the Wigner function is given by $W\left(\beta, \beta^{*}\right)=2 \exp \left(-2|\beta-\alpha|^{2}\right) / \pi$ so that the corresponding probability distribution of balanced homodyne photodetection is given by

$$
P_{\theta_{L}}\left(q_{\theta_{L}}\right)=\frac{1}{\sqrt{\pi}} \exp \left(-\left(q_{\theta_{L}}-\tilde{q}_{\theta_{L}}\right)^{2}\right)
$$

with $\tilde{q}_{\theta_{L}}=\frac{1}{\sqrt{2}}\left(\alpha e^{-i \theta_{L}}+\alpha^{*} e^{i \theta_{L}}\right)$. Thus, postselecting photon counts by balanced homodyne photodetection with difference photon numbers $n_{-}$in the range $n_{-} / \sqrt{2}\left|\alpha_{L}\right| \in\left(\tilde{q}_{\theta_{L}}-\delta_{L}, \tilde{q}_{\theta_{L}}+\delta_{L}\right)$ is equivalent to projection onto the coherent state $|\alpha\rangle$ with probability

$$
\operatorname{Prob}(\alpha)=\operatorname{erf}\left(\delta_{L}\right) \geq 1-\frac{e^{-\delta_{L}^{2}}}{\sqrt{\pi} \delta_{L}}
$$

with $\operatorname{erf}(x)$ denoting the error function [34]. Thus, choosing $\delta_{L}=2$, for example, yields $\operatorname{Prob}(\alpha)>0.9953222650$ and $\delta_{L}=3$ yields $\operatorname{Prob}(\alpha)>0.9999779095$.

\section{Appendix C: Time evolution of different qubits}

In this appendix we analyze the more general situation when the qubits have different coupling strengths to the cavity and also different transition frequencies. We focus on small deviations from the ideal Hamiltonian in Eq. (11) which in this case is replaced by

$\hat{H}=\hbar \omega \hat{a}^{\dagger} \hat{a}+\sum_{i=A, B} \hbar\left(\frac{\delta_{i}-\omega}{2} \hat{\sigma}_{i}^{z}+g_{i} \hat{\sigma}_{i}^{+} \hat{a}+g_{i} \hat{\sigma}_{i}^{-} \hat{a}^{\dagger}\right)$

where $g_{A}=g+\varepsilon_{g}, g_{B}=g-\varepsilon_{g}, \delta_{A}=\delta+\varepsilon_{\delta}$ and $\delta_{B}=$ $\delta-\varepsilon_{\delta}$. The state $\left|\Psi^{-}\right\rangle|n\rangle$ is no longer an eigenstate of the Hamiltonian in Eq. (C1). Therefore, for photon numbers $n \geq 2$ its blocks are $4 \times 4$ matrices which in the basis $\left\{\left|\psi^{-}\right\rangle|n-1\rangle,|1,1\rangle|n-2\rangle,\left|\Psi^{+}\right\rangle|n-1\rangle,|0,0\rangle|n\rangle\right\}$ are given by

$H^{(n \geq 2)}=$

$\hbar\left(\begin{array}{cccc}\omega(n-1) & \varepsilon_{g} \sqrt{2 n-2} & -\varepsilon_{\delta} & -\varepsilon_{g} \sqrt{2 n} \\ \varepsilon_{g} \sqrt{2 n-2} & \omega(n-1)+\delta & g \sqrt{2 n-2} & 0 \\ -\varepsilon_{\delta} & g \sqrt{2 n-2} & \omega(n-1) & -g \sqrt{2 n} \\ \varepsilon_{g} \sqrt{2 n} & 0 & -g \sqrt{2 n} & \omega(n-1)-\delta\end{array}\right)$.

Thereby, for the sake of simplicity we have concentrated to the special case of $\theta=0$ so that the coupling strengths $g$ are positive. However, the eigenvalues of the matrices of Eq. C2 do not depend on the choice of this phase.

If $\delta \ll \omega_{\bar{n}}$ and the number of excitations is large, i.e. $n>>1$, the four eigenvalues of each block are approximately given by the two pairs $\hbar \omega(n-1) \pm \hbar \Omega_{n}^{(\mathrm{S})}$ and $\hbar \omega(n-1) \pm \hbar \Omega_{n}^{(\mathrm{L})}$. They reduce to $\hbar \omega(n-1) \pm 0$ and $\hbar \omega(n-1) \pm \hbar \Omega_{n}$ in the limit of $\epsilon_{g} \rightarrow 0$ and $\epsilon_{\delta} \rightarrow 0$. Therefore, the asymmetries between the coupling strengths and the detunings induce new Rabi oscillations that will collapse (and revive) at a slower time scale. This behaviour can be identified in Fig. 6. To estimate this time scale we aim for a coherent state expansion of the photonic state $\left|\chi_{2}(t)\right\rangle$ involving the smallest frequencies. In order to obtain simple analytical solutions that approximate the eigenvalues of (C2) we take the zeroth order expansion analogue to Eq. A3 . Linearizing the pair of smallest eigenvalues in $\varepsilon_{g}, \varepsilon_{\delta}$ and $n-\bar{n}$ we obtain $\Omega_{n}^{(\mathrm{S})} \approx \Delta+\varpi(n-\bar{n})$ with

$$
\begin{aligned}
& \Delta=\frac{\omega_{\bar{n}}^{2}}{g \Omega_{\bar{n}}} \varepsilon_{g}+\frac{\delta}{\Omega_{\bar{n}}} \varepsilon_{\delta}, \\
& \varpi=2 \frac{\Omega_{\bar{n}}^{2}+\delta^{2}}{\Omega_{\bar{n}}^{3}} g \varepsilon_{g}+\frac{g^{2} \delta}{\Omega_{\bar{n}}^{3}} \varepsilon_{\delta} .
\end{aligned}
$$

The field state that accompanies the Bell state $\left|\Psi^{-}\right\rangle$in Eq. (28) can be assumed to depend on coherent states in a similar way as the states in Eq. (9) and therefore can be written in the form

$$
\left|\chi_{2}(t)\right\rangle \approx \sum_{ \pm} \eta_{2, \pm} \mathrm{e}^{\mp i(\Delta-\varpi \bar{n}) t}\left|\alpha \mathrm{e}^{-i(\omega \pm \varpi) t}\right\rangle .
$$

In the case of small values of $\varepsilon_{\delta}$ and $\varepsilon_{g}$ the deviation of the state $\left|\chi_{2}(t)\right\rangle$ from the coherent state evolving with frequency $\omega$ is small. The exact form of the coefficients in Eq. (C4) is not relevant for our analysis as we focus only on the frequencies of the system. From Eq. (11) we can conclude that the overlap $\left|\left\langle\alpha \mathrm{e}^{-i \omega t} \mid \chi_{2}(t)\right\rangle\right|^{2}$ undergoes Rabi oscillations at frequency $2 \Delta$ which decay as $\exp \left\{-\bar{n} \varpi^{2} t^{2}\right\}$.

At this point it is convenient to summarize the steps of our scheme in order to have a clear picture of how the fidelity of the final state and the success probability of post-selecting the state $\left|\Psi^{-}\right\rangle$change. We start with the initial state of Eq. (2) which for an interaction time $\tau$ evolves under the influence of the Hamiltonian in Eq. (C1) to a state given by Eq. (28). A projection onto the photonic state $\left|\alpha \mathrm{e}^{-i \omega \tau}\right\rangle$ is performed with success 
probability $P=\sum_{j=-1}^{2}\left|z_{j}\right|^{2}$ which is given in terms of the overlaps $z_{j}=\left\langle\alpha \mathrm{e}^{-i \omega \tau} \mid \chi_{j}(\tau)\right\rangle$. In the ideal case this reduces to the expression of Eq. (17). The resulting material qubits are allowed to interact with a second cavity prepared in a coherent state that differs from the first coherent state by a phase $\tilde{\varphi}$ given in Eqs. (23) and (26). Thereby, the tripartite system for the second interaction is given by

$$
\left|\Psi_{1}\right\rangle=\frac{1}{\sqrt{P}}\left(z_{2}\left|\Psi^{-}\right\rangle+z_{1}|1,1\rangle+z_{0}\left|\Psi^{+}\right\rangle+z_{-1}|0,0\rangle\right)\left|\alpha \mathrm{e}^{i \tilde{\varphi}}\right\rangle .
$$

This state evolves under the action of a Hamiltonian $\hat{H}^{\prime}$ in the form of Eq. (C1) to the state $\left|\Psi^{\prime}(\tau)\right\rangle$ in the form of Eq. (28) with the photonic states $\left|\chi_{j}^{\prime}(\tau)\right\rangle, j=-1,0,1,2$. A projection onto the field state $\left|\alpha \mathrm{e}^{i(\tilde{\varphi}-\omega \tau)}\right\rangle$ is performed with success probability $P^{\prime}=\sum_{j=-1}^{2}\left|z_{j}^{\prime}\right|^{2}$ with $z_{j}^{\prime}=\left\langle\alpha \mathrm{e}^{i(\tilde{\varphi}-\omega \tau)} \mid \chi_{j}^{\prime}(\tau)\right\rangle$. The total success probability of the scheme is given by $P_{T}=P P^{\prime}$ and the material qubits result in the state

$$
\left|\psi_{f}\right\rangle=\frac{1}{\sqrt{P^{\prime}}}\left(z_{2}^{\prime}\left|\Psi^{-}\right\rangle+z_{1}^{\prime}|1,1\rangle+z_{0}^{\prime}\left|\Psi^{+}\right\rangle+z_{-1}^{\prime}|0,0\rangle\right)
$$

which in the ideal case matches the Bell state $\left|\Psi^{-}\right\rangle$. The fidelity with respect to this Bell state is $F=\left|\left\langle\Psi^{-} \mid \psi_{f}\right\rangle\right|$ and in the ideal case it attains values close to unity.
In our numerical examples the success probability involves two measurements at time $\tau \rightarrow \tau_{r} / 4$ with the revival time $\tau_{r}$ as given by Eq. (12). Its decay depends on $\varpi$ and it is proportional to $\exp \left\{-2 \bar{n} \varpi^{2}\left(\tau_{r} / 4\right)^{2}\right\}$. This sets a boundary for the applicability of our scheme as the success probabilities become arbitrarily small for large arguments of the exponential function. The boundary for which the argument of the exponential is less than unity is determined by the inequality

$$
\frac{\varepsilon_{g}}{g} \leq \frac{1}{\pi} \sqrt{\frac{2}{\bar{n}}}\left(1+\frac{\delta^{2}}{\Omega_{\frac{2}{n}}}\right)^{-1}-\frac{1}{2} \frac{\delta \varepsilon_{\delta}}{\Omega_{\frac{2}{n}}^{2}+\delta^{2}}
$$

The oscillations of the fidelity in Fig. 7 achieve their maximum values close to unity whenever the asymmetry $\varepsilon_{g}$ is such that the corresponding Rabi oscillation completes a cycle at the interaction time $\tau=\tau_{r} / 4$. This condition is fulfilled for $2 \Delta \tau_{r} / 4=2 l \pi$ with $l \in \mathbb{N}_{+}$. From Eq. (C3) we obtain that this happens for

$$
\varepsilon_{g}^{(l)} / g=\left(4 g^{2} l-\delta \varepsilon_{\delta}\right) / \omega_{n}^{2}
$$

which is taken for a fixed value of $\varepsilon_{\delta}$. The first minimum in the fidelity as a function of $\varepsilon_{g}$ occurs at $\varepsilon_{g}^{(1)} / 2$. For $\varepsilon_{\delta}=0$ this value is approximately $1 / 2 \bar{n}$.
[1] M. A. Nielsen and I. L. Chuang, Quantum Computation and Quantum Information (Cambridge, Univ.Press, 2000).

[2] H. J. Kimble, Nature(London) 453, 1023 (2008).

[3] C. H. Bennett, G. Brassard, C. Crépeau, R. Jozsa, A. Peres, and W. K. Wootters, Phys. Rev. Lett. 70, 1895 (1993).

[4] N. Sangouard, C. Simon, H. de Riedmatten, and N. Gisin, Rev. Mod. Phys. 83, 33 (2011).

[5] M. Zukowski, A. Zeilinger, M. A. Horne, and A. K. Ekert, Phys. Rev. Lett. 71, 4287 (1993).

[6] H. de Riedmatten, I. Marcikic, J. A. W. van Houwelingen, W. Tittel, H. Zbinden, and N. Gisin, Phys. Rev. A 71, 050302 (2005).

[7] M. Halder, A. Beveratos, N. Gisin, V. Scarani, C. Simon, and H. Zbinden, Nature Phys. 3, 692 (2007).

[8] Y. Xue, A. Yoshizawa, and H. Tsuchida, Phys. Rev. A 85, 032337 (2012).

[9] W. Dür, H.-J. Briegel, J. I. Cirac, and P. Zoller, Phys. Rev. A 59, 169 (1999).

[10] Z. Zhao, T. Yang, Y.-A. Chen, A.-N. Zhang, and J.-W. Pan, Phys. Rev. Lett. 90, 207901 (2003).

[11] R. Reichle, D. Leibfried, E. Knill, J. Britton, R. B. Blakestad, J. D. Jost, C. Langer, R. Ozeri, S. Seidelin, and D. J. Wineland, Nature(London) 443, 838 (2006).

[12] D. Boschi, S. Branca, F. De Martini, L. Hardy, and S. Popescu, Phys. Rev. Lett. 80, 1121 (1998).

[13] D. Bouwmeester, J.-W. Pan, K. Mattle, M. Eibl, H. Weinfurter, and A. Zeilinger, Nature (London) 390, 575 (1997).

[14] A. Furusawa, J. L. Sørensen, S. L. Braunstein, C. A.
Fuchs, H. J. Kimble, and E. S. Polzik, Science 282, 706 (1998).

[15] J. Yin et al., Nature (London) 488, 185 (2012).

[16] X.-S. Ma et al., Nature (London) 489, 269 (2012).

[17] M. Riebe, H. Häffner, C. F. Roos, W. Hänsel, J. Benhelm, G. P. T. Lancaster, T. W. Körber, C. Becher, F. SchmidtKaler, D. F. V. James, and R. Blatt, Nature (London) 429, 734 (2004).

[18] M. D. Barrett, J. Chiaverini, T. Schaetz, J. Britton, W. M. Itano, J. D. Jost, E. Knill, C. Langer, D. Leibfried, R. Ozeri, and D. J. Wineland, Nature (London) 429, 737 (2004).

[19] C. Nölleke, A. Neuzner, A. Reiserer, C. Hahn, G. Rempe, and S. Ritter, Phys. Rev. Lett. 110, 140403 (2013).

[20] P. van Loock, T. D. Ladd, K. Sanaka, F. Yamaguchi, K. Nemoto, W. J. Munro, and Y. Yamamoto, Phys. Rev. Lett. 96, 240501 (2006).

[21] M. Tavis and F. W. Cummings, Phys. Rev. 170, 379 (1968).

[22] W. P. Schleich Quantum Optics in Phase Space (WileyVCH, Weinheim, 2001).

[23] A. I. Lvovsky and M. G. Raymer, Rev. Mod. Phys. 81, 299 (2009).

[24] We assume that the state of the single mode resonator can be perfectly transferred into a single signal mode to be measured by balanced homodyne photodetection.

[25] K. Vogel and H. Risken, Phys. Rev. A 40, 2847 (1989).

[26] D. A. Rodrigues, C. E. A. Jarvis, B. L. Györffy, T. S. Spiller, and J. F. Annett, J. Phys.:Condens. Matter 20 075211 (2008).

[27] S. Gleyzes, S. Kuhr, C. Guerlin, J. Bernu, S. Deléglise, 
U. B. Hoff, M. Brune, J.-M. Raimond, and S. Haroche, Nature 446, 297 (2007).

[28] J. Z. Bernád and G. Alber, Phys. Rev. A 87, 012311 (2013).

[29] D. Gonta and P. van Loock, Phys. Rev. A 86, 052312 (2012).

[30] B. Casabone, A. Stute, K. Friebe, B. Brandstätter, K. Schüppert, R. Blatt, and T. E. Northup, Phys. Rev. Lett. 111, 100505, (2013).
[31] Y. Colombe, T. Steinmetz, G. Dubois, F. Linke, D Hunger, and J. Reichl, Nature 450, 272 (2007).

[32] J. M. Torres, E. Sadurni, and T. H. Seligman, J. Phys. A 43, 192002 (2010).

[33] P. L. Kelly and W. H. Kleiner, Phys. Rev. 136, A316 (1964).

[34] Handbook of Mathematical Functions, M. Abramowitz and I. A. Stegun eds. (Dover, N.Y., 1965). 